\title{
GCU
}

Glasgow Caledonian

University

University for the Common Good

\section{A continuum model of stresses in a vertical silo with a flow channel in the vicinity of the wall, using the principal stress cap surface approach for the bulk solids}

Matchett, A.J.; Langston, P.A.; McGlinchey, D.

Published in:

Chemical Engineering Research and Design

DOI:

10.1016/j.cherd.2017.04.013

Publication date:

2017

Document Version

Author accepted manuscript

Link to publication in ResearchOnline

Citation for published version (Harvard):

Matchett, AJ, Langston, PA \& McGlinchey, D 2017, 'A continuum model of stresses in a vertical silo with a flow channel in the vicinity of the wall, using the principal stress cap surface approach for the bulk solids', Chemical Engineering Research and Design, vol. 122, pp. 211-225. https://doi.org/10.1016/j.cherd.2017.04.013

\section{General rights}

Copyright and moral rights for the publications made accessible in the public portal are retained by the authors and/or other copyright owners and it is a condition of accessing publications that users recognise and abide by the legal requirements associated with these rights.

Take down policy

If you believe that this document breaches copyright please view our takedown policy at https://edshare.gcu.ac.uk/id/eprint/5179 for details of how to contact us. 
A continuum model of stresses in a vertical silo with a flow channel in the vicinity of the wall, using the principal stress cap surface approach for the bulk solids

\section{A.J.Matchett, University of Teesside(retired) ; P.A..Langston, Faculty of Engineering, University of Nottingham; D.McGlinchey, School of Engineering, Glasgow Caledonian University}

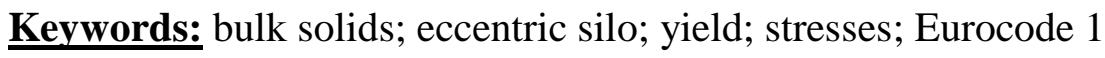

\section{Summary}

Eurocode 1(2006) gives design equations for eccentric stresses in silos, including flow channels adjacent to the wall. This has been modelled using the approach of Matchett et al(2015, 2016).

A three zone model was developed, consisting of:

- the flow channel

- the transition zone

- the bulk of the solids

The flow channel and the transition zone were modelled by Janssen-type equations. The bulk was modelled by the principal stress cap approach.

The transition zone is a complex region and has several purposes:

1. to shelter the low stress flow channel from the high stresses around

2. to allow high principal stresses at the transition/bulk interface, within the yield locus

3. to form a transition between the dynamic flow channel and the static bulk

4. to allow transition from passive stress in the flow channel to active stress in the bulk

The model was calibrated against the data of Chen et al(2007) for a full-scale silo, and described the data reasonably well, scaling axially and azimuthally.

Large experimental data sets are required to calibrate a model. Unfortunate data points cannot be arbitrarily rejected.

Further extensive, experimental data are needed to calibrate models. 


\section{Introduction}

Matchett, Langston and McGlinchey $(2015,2016)$ describe how eccentric stresses in vertical, cylindrical silos were modelled. They used a "principal stress cap surface" approach, in which the geometry of the principal stress cap and principal stress paths in 3-dimensional space were assumed "a priori" - see Figure 1.

Seen from above, the principal stresses in the azimuthal direction describe circular stress paths in the horizontal plane.

In a symmetrical system, the circle would be centred about the centre of the silo, of radius $r_{2}$, creating a series of concentric circles. In an asymmetrical system, the centre of the core, radius $r_{1}$, was displaced a distance $h$, causing the circular stress paths to be compacted in the direction of displacement and expanded in the opposite direction. The centre of the core is now the centre of stress, and a Cartesian axis was defined at this point, with $\mathrm{x}$ in the direction of displacement and z vertically. The centres of successive, azimuthal principal stress circles were also displaced, and a given circle can be characterised by the value $\mathrm{X}$ (upper case $\mathrm{x}$ ), its value at the point where it crosses the $\mathrm{x}$ axis.

The vertical height was characterised by parameter $\mathrm{Z}$ (upper case $\mathrm{z}$ ). This is the value of $\mathrm{z}$ at a characteristic point on the principal cap surface - in this case the value of $\mathrm{z}$ at the core. The principal stress cap surface describes a circular arc in the $\mathrm{x}-\mathrm{z}$ plane, along the $\mathrm{x}$-axis, with characteristic radii $R_{20}$ in the positive sense of $x$ and $R_{2 \pi}$ in the negative direction of $x$. $A$ given circle subtends an angle $\varepsilon_{2}$ to the vertical plane. $\varepsilon_{2}$ is constant around a line of constant $\mathrm{X}$. Thus, an $\mathrm{X}$-line describes the path of principal stress $\sigma_{2}$ and is a circular projection onto the horizontal plane of an elliptical path in 3 dimensions.

On the surface of the principal stress cap and normal to the X-lines, are the "radial" stress paths of stress $\sigma_{1}$, given by the $\psi_{1}$-lines. A projection onto the horizontal plane is shown in Figure 2.

The principal stress $\sigma_{3}$ is normal to the stress cap surface - Figure 1, lower section. Its path in space is described by $\psi_{2}$ lines, normal to the principal stress cap surface.

A given $\mathrm{X}$-line is at an angle of $\lambda$ to the vertical, when seen from the $\mathrm{y}$-axis. At a given point $\mathrm{P}$, at rotation angle $\varepsilon_{1}$ is inclined at angle $\varepsilon_{3}$.

All relevant parameters to describe the surface can be deduced from the preceding assumptions: Matchett et al $(2015,2016)$

Genuine 3-dimensional force balances were performed in principal stress space, based upon an incremental element bounded by $X, \psi_{1}$ and $\psi_{2}$ lines, to determine stress distributions 
within the silo. This gives orthogonal equations in $\sigma_{1}, \sigma_{2}$ and $\sigma_{3}$ : see Figure 1 , lower section for local principal stress axes.

The methodology is a true 3-d development of the concept of the principal stress cap first proposed by Enstad: Enstad(1975), Nedderman(1992). It carries forward many of the advantages and limitations of the Enstad approach:

- The models are steady-state. They can only model static systems or systems at incipient flow

- The bulk materials are modelled as rigid-plastic solids: there are no elastic components

- The model applies to the central, axial section of a silo (preferably a slender silo), in which the stresses are fully developed, as opposed to the surface where stresses are affected by the surface interface and any surcharge, or the base where the geometry of the silo bottom affects stress patterns

- The models are relatively simple, both conceptually and numerically, when compared to DEM, FEM and other true 3-d models of bulk solids in silos

- The models predict stress distributions from assumed principal stress orientations. The numerical algorithms are relatively simple and quick, and model outputs can be obtained in tens of minutes, compared to days for some, more sophisticated algorithms

- Post-calculation the validity of the outputs must be tested by some means: Conical Yield Function (CYF) and Wall Yield Function (WYF) were used to assess the validity of model outputs: Matchett et al(2016)

- The models give extensive 3-dimensional data for stress distributions within silos, as well as wall stress data

The following findings were inferred from the simulation data:

1. Completely filled silos, (Matchett et al, 2015) and silos with finite cores (Matchett et al, 2016) were modelled

2. There are at least two types of stress eccentricity in silos: surcharge or surface eccentricity, and inherent eccentricity: Matchett et al(2015)

3. Surcharge eccentricity is due to an uneven distribution of surcharge material on top of an underlying, uniform stress pattern. Eccentricities quickly decay with depth, as per the Janssen model( Jannsen, 1895) 
4. Inherent eccentricity is due to an underlying, off-centre centre-of-stress. Stress eccentricities increase with depth

5. The models were able to predict wall stresses corresponding to a DEM simulation: Matchett et al(2015)

6. A range of internal stress parameters within the model were able to give reasonable approximations to the DEM wall stress output. There were no indications of a unique relationship between internal structure and wall stress distribution, and a range of parameters give reasonable approximations to external data.

7. Large variations in wall normal stress were found at high eccentricities and large wall angles. However, such systems were often found to be non-viable as they exceeded the Yield Loci

8. Expected features of silo behaviour were modelled, including: passive(convex stress cap) systems; active(concave stress cap) systems; incipient flow and switch stress: Matchett et al(2016)

9. Two recurrent themes in the papers were: the need for experimental data for internal stress distributions in silos; the inability to predict internal stress distributions from wall stress measurements

The systems modelled consisted of eccentric cores which did not extend beyond the limits of the silo wall.

Many workers have studied systems where there is a flow channel that touches the silo wall, as shown in Eurocode 1(2006) - Section 5.2.4.3, page 52, Figure 5.5. Other workers have studied systems where the flow channel is in the region of the silo wall, including Sielamovicz et el(2010, 2011, 2015), Chen et al(2007) and Lapko(2010).

This paper seeks to extend the 3-d, principal stress cap technique to cover situations of wall discharge approximating to the Eurocode 1 analysis.

\section{The Eurocode 1 silo design algorithm for a flow channel in the vicinity of the silo wall}

The Eurocode 1 design standard for silos presents a series of equations for the calculation of wall normal stresses for the purposes of structural design of the silo.

The code proposes 3 zones:

- A flowing zone, in which the material is in motion to discharge down the silo

- A static zone far(relatively) from the flowing zone, where the solids remain static

- A high stress zone, between the flow channel zone and the bulk zone 
$\theta_{c}$ is the half-angle subtended between the edge of the flow zone, the silo centre, and the centre of the flow zone. The high stress zone is also assumed to be of angle $\theta_{c}$ beyond the flow channel. The remainder of the silo is assumed to be in the static zone. The code presents equations to calculate wall normal and vertical stresses in each zone. A typical calculation is shown in Figure 3 for the material used in example calculations in previous papers: Matchett et $\mathrm{al}(2015,2016)$. The static bulk material has an intermediate magnitude of stress, the high stress zone is the largest stress, and the flow zone has a relatively low stress. This analysis is very stark compared with the experimental data of Chen et al(2007), but the existence of the high stress zone has been shown by many workers, for example Chen et al(2007) and Lapko(2010).

\section{A two-zone model for a flow channel in the vicinity of the silo wall}

An initial consideration of the situation of a wall flow channel suggests that there are two zones, Figure 4, Sielamovicz et al(2010, 2011), Eurocode 1(2006), Figure 5.5a:

- The flow channel, adjacent to the wall

- The remainder of the silo, containing bulk solids

For the purposes of this modelling exercise, it will be assumed that a region of fully developed stress and steady state flow exists between the surface and the bottom of the silo. This is the region that will be modelled. An equivalent approach was taken in the previous papers(Matchett et al, 2015, 2016).

Consider a horizontal slice through the region of fully developed stress - Figure 5. The silo has radius $r_{2}$ and centre at $\mathrm{O}_{2}$. The silo wall is cut by the flow channel, which is of circular cross-section and radius $r_{1}$. The flow channel section is assumed to cut the silo wall at rightangles. The silo wall is a projected path of circumferential(azimuthal) principal stress, $\sigma_{2}$. Hence, the flow channel wall is a projected path of principal stress $\sigma_{1}$. The point of intersection projects a half-angle $\theta_{\mathrm{o}}$ at the silo centre, and has half-chord length $1_{\mathrm{o}}$. The normality of the silo and flow channel is contrary to Eurocode 1, where the intersection angle is determined by a minimum flow area basis. However, normality is essential in order to implement the principal stress cap model to the bulk zone.

A reference set of Cartesian axes is placed $(x, y, z)$ is placed at the apex of the flow channel border, along the line connecting the two circle centres, with $\mathrm{x}$ and $\mathrm{y}$ in the horizontal plane and $z$ vertically out of the plane. 
As in the previous models(Matchett et al 2015, 2016), it is assumed that the azimuthal principal stress, $\sigma_{2}$, projects a circular path onto the horizontal plane. A generalised path is shown in Figure 6. The circle has an intercept X(upper case) with the reference X-axis and a radius $\mathrm{R}_{1}$. The general stress path circle cuts the flow channel border normally. The circle has a centre $\mathrm{O}$. The half-angle between the circle centre and the point of intersection with the flow channel wall is $\theta$, with half-chord length 1 .

A general point $\mathrm{P}$ - Figure 6 , can be identified by specifying the circle on which it sits, through its intersection $X$, and the subtended angle between the arc and circle centre $O, \varepsilon_{1}$. The third co-ordinate is provided by $\mathrm{Z}$, the intersection of the characteristic location of the principal stress cap with the z-axis: Matchett et al(2015, 2016).

Within the silo, the $\mathrm{X}$-lines cut the $\mathrm{x}$-axis at various values of $\mathrm{x}$, to give the $\mathrm{X}$ value of each projected circle. These plot the course of azimuthal stress $\sigma_{2}$. The X-lines are crossed by $\psi_{1}$-lines, which mark the paths of principal stress $\sigma_{1}$ - Figure 7. Each $\psi_{1}$ - line has a unique, identifying value marked by the value of angle $\psi_{1}$ at the flow channel border wall of $\psi_{10}$. The flow channel border is therefore, also a $\psi_{1}$-line and marks the limit of the principal stress cap system.

The stress and co-ordinate system is a direct development of the models presented in the previous papers(Matchett et al 2015, 2016), with geometric modifications. The equations from Matchett et al(2015) can be directly transposed to the present system, taking account of geometrical differences. Therefore, this model will be presented in outline only, with emphasis on those changes from the original model. The remainder of the parameters are as in the original model and original notation is included in this paper for convenience.

The reader is referred to Matchett et al(2015) for details.

\section{$\mathrm{X}$-line geometry}

The silo wall, radius $r_{2}$ and the flow channel wall, radius $r_{1}$ cross normally. It can be shown that:

$$
\begin{aligned}
& \sin \theta_{o}=\frac{l_{o}}{r_{2}} \\
& r_{1}=r_{2} \tan \theta_{o}
\end{aligned}
$$

And for a general X-circle with subtended half-angle $\theta$ :

$$
\begin{aligned}
& l=R_{1} \sin \theta=r_{1} \cos \theta \\
& R_{1}=r_{1} \cot \theta
\end{aligned}
$$


and $\theta$ is related to co-ordinate system variable $\varepsilon_{1}$ or $\psi_{1}$ by:

$$
\varepsilon_{1}=\pi-\theta
$$

The value of $\mathrm{X}$ is given by:

$$
X=R_{1}(1+\cos \theta)-r_{1}(1-\sin \theta)
$$

The co-ordinate of the centre of circle $\mathrm{X}, \mathrm{O}$ :

$$
O=R_{1} \cos \theta-r_{1}(1-\sin \theta)
$$

At the silo wall:

$$
\begin{aligned}
& R_{1}=r_{2}: X=X_{\max }: \theta=\theta_{o} \\
& X_{\text {max }}=r_{2}(1+\cos \theta)-r_{1}(1-\sin \theta)
\end{aligned}
$$

The path of a given $\psi_{1}$-line is described by two parameters: $a_{1}$ and $a_{2}$ :

$$
\begin{aligned}
& a_{1}=\frac{\partial R_{1}}{\partial X} \\
& a_{2}=\frac{\partial O}{\partial X}
\end{aligned}
$$

such that:

$$
\begin{aligned}
& \frac{\partial w_{1}}{\partial X}=a_{1}+a_{2} \cos \psi_{1}=a_{1}+a_{2} \cos \varepsilon_{1} \\
& \frac{\partial \psi_{1}}{\partial X}=\frac{a_{2} \sin \psi_{1}}{R_{1}}=\frac{a_{2} \sin \varepsilon_{1}}{R_{1}}
\end{aligned}
$$

hence:

$$
a_{1}=\frac{\partial R_{1}}{\partial X}=-r_{1}^{2} \operatorname{cosec}{ }^{2} \theta \frac{\partial \theta}{\partial X}
$$

and

$$
\begin{aligned}
& a_{2}=\frac{\partial O}{\partial X}=\left\{-R_{1} \sin \theta+\cos \theta \frac{\partial R_{1}}{\partial \theta}+r_{1} \cos \theta\right\} \frac{\partial \theta}{\partial X} \\
& \frac{\partial X}{\partial \theta}=(1+\cos \theta) \frac{\partial R_{1}}{\partial \theta}-R_{1} \sin \theta+r_{1} \cos \theta \\
& \frac{\partial R_{1}}{\partial \theta}=-r_{1} \operatorname{cosec}{ }^{2} \theta
\end{aligned}
$$

The previous models had $\mathrm{a}_{1}$ and $\mathrm{a}_{2}$ as constants. In this version, $\mathrm{a}_{1}$ and $\mathrm{a}_{2}$ are variables and functions of $\theta$ and hence $X$.

The surface geometry of the principal stress cap 
Figure 8 shows a represenation of the principal stress cap surface including a plan view and view from the $y$-axis. Seen from the $y$-axis, the total surface is represented by ABC. AB is a circular arc along and above the $\mathrm{x}$-axis. $\mathrm{BC}$ is the $\lambda$-line at the silo wall. ADE is the portion of the stress cap within the circle $\mathrm{X}$, where DE is the corresponding $\lambda$-line.

Reference co-ordinate $\mathrm{Z}$ is the height of the apex of the principal stress cap/flow channel internal perimeter at $\mathrm{x}=0: \mathrm{y}=0$

Line $\mathrm{AB}$, along the $\mathrm{x}$-axis, is assumed to be a circular arc in the $\mathrm{x}-\mathrm{z}$ plane. A given point, $\mathrm{F}$, subtends an angle $\varepsilon_{2}$ with the vertical. The value of $\varepsilon_{2}$ is constant around the X-circle, and $\varepsilon_{2}$ is a function of $\mathrm{X}$ only.

$\mathrm{X}=0$ is the centre of stress for the bulk, principal stress cap system. Symmetry is assumed, as in the Virtual Core of the previous model(Matchett et al, 2015). Therefore, at $X=0 ; \varepsilon_{2}=0$, or in terms of the previous model: $\beta_{\mathrm{c}}=0$

$\lambda$ is the slope of the $\mathrm{X}$-line as seen from the $\mathrm{y}$-axis. It is a projection of the path around the $\mathrm{X}$ line in 3-d space. The sense of the slope of the $\lambda$-line is the opposite of the original model (Matchett et al 2015). The slope is downwards with increasing $\mathrm{x}$ in this model, whereas it was upwards with $\mathrm{x}$ in Matchett et al(2015, 2016).

It is assumed that the $\psi_{1}$-line at the flow channel border describes a circular arc on the unrolled, flattened surface of the border. It has a length $\mathrm{P}_{\mathrm{f}}$ : the internal half perimeter of the flow channel:

$$
P_{f}=r_{1}\left(\pi / 2-\vartheta_{o}\right)
$$

The circular arc progresses across the flow channel perimeter from $\varepsilon_{2}=0$ to $\varepsilon_{2}=\beta_{\mathrm{w}}$, the wall normal angle. The arc has a radius $\mathrm{R}_{2 \theta}$, where:

$$
R_{2 \theta}=\frac{P_{f}}{\left(\sin \beta_{w}-\sin \beta_{c}\right)}=\frac{P_{f}}{\sin \beta_{w}}
$$

Thus, for a given value of $\mathrm{X}$ and therefore $\varepsilon_{2}$, the distance of the intersection below reference level $\mathrm{Z}, \mathrm{dZ}_{\theta}$ is:

$$
d Z_{\theta}=R_{2 \theta}\left(\cos \beta_{c}-\cos \varepsilon_{2}\right)=R_{2 \theta}\left(1-\cos \varepsilon_{2}\right)
$$

Likewise, the $\mathrm{x}$-direction displacement in negative $\mathrm{x}$-space, $\mathrm{dX} \mathrm{X}_{\theta}$ is given by:

$$
d X_{\theta}=r_{1}(1-\sin \theta)
$$

The geometry underpinning the calculation of $\lambda$ is shown in Figure 9. 


$$
\tan \lambda=\frac{d Z-d Z_{\theta}}{X+d X_{\theta}}
$$

The $\left(\varepsilon_{1}, \mathrm{X}, \mathrm{Z}\right)$ co-ordinate system can then be related to the $(\mathrm{x}, \mathrm{y}, \mathrm{z})$ system as follows:

$$
\begin{aligned}
& x=O+R_{1} \cos \varepsilon_{1} \\
& y=R_{1} \sin \varepsilon_{1} \\
& z=Z-\left(x+d X_{\theta}\right) \tan \lambda-d Z_{\theta}
\end{aligned}
$$

A typical principal stress cap surface is shown in Figure 10.

\section{Force Balances}

The changes in geometry are taken into account in the 3 force balance equations(Matchett et al 2015, 2016). The change in sense of $\lambda$ and hence $\varepsilon_{3}$ results in changes in sign of some of the terms in the equations, but fundamentally, the equations are as in the previous model:

$$
\begin{aligned}
& \frac{\partial}{\partial \varepsilon_{1}}\left[\sigma_{2} R_{2} \frac{\partial \varepsilon_{2}}{\partial X} \frac{\partial w_{2}}{\partial Z}\right]= \\
& -\sigma_{1} \frac{R_{1}}{\cos \varepsilon_{3}} \frac{\partial w_{2}}{\partial Z} E \frac{\partial \psi_{1}}{\partial X}+\sigma_{3}\left(\frac{R_{1}}{\cos \varepsilon_{3}}\right) R_{2} \frac{\partial \varepsilon_{2}}{\partial X} \frac{D \varepsilon_{3}}{D Z}-\rho g R_{1} R_{2} \frac{\partial \varepsilon_{2}}{\partial X} \frac{\partial w_{2}}{Z} \cos \varepsilon_{2} \tan \varepsilon_{3}
\end{aligned}
$$

$$
\begin{aligned}
& \frac{D}{D X}\left[\sigma_{1} \cos \varepsilon_{2}\right]= \\
& -\frac{\sigma_{1}}{R_{1} \cos \varepsilon_{3}}\left(\frac{\partial w_{1}}{\partial X}\right)\left(\frac{\partial w_{2}}{\partial Z}\right)+\sigma_{2}\left(\frac{R_{2}}{R_{1}}\right) E \frac{\partial \varepsilon_{2}}{\partial X} \frac{\partial w_{2}}{\partial Z}-\sigma_{3}\left(\frac{R_{2}}{\cos \varepsilon_{3}}\right) \frac{\partial \varepsilon_{2}}{\partial X} \frac{D \varepsilon_{2}}{D Z}+\rho g R_{2} \frac{\partial \varepsilon_{2}}{\partial X} \frac{\partial w_{2}}{\partial Z} \sin \varepsilon_{2}
\end{aligned}
$$

or

$$
\begin{gathered}
\frac{\partial}{\partial X}\left[\sigma_{1} \cos \varepsilon_{2}\right]=-\frac{\partial}{\partial \varepsilon_{1}}\left[\sigma_{1} \cos \varepsilon_{2}\right] \frac{\partial \psi_{1}}{\partial X} \\
-\frac{\sigma_{1}}{R_{1} \cos \varepsilon_{3}}\left(\frac{\partial w_{1}}{\partial X}\right)\left(\frac{\partial w_{2}}{\partial Z}\right)+\sigma_{2}\left(\frac{R_{2}}{R_{1}}\right) E \frac{\partial \varepsilon_{2}}{\partial X} \frac{\partial w_{2}}{\partial Z}-\sigma_{3}\left(\frac{R_{2}}{\cos \varepsilon_{3}}\right) \frac{\partial \varepsilon_{2}}{\partial X} \frac{D \varepsilon_{2}}{D Z}+\rho g R_{2} \frac{\partial \varepsilon_{2}}{\partial X} \frac{\partial w_{2}}{\partial Z} \sin \varepsilon_{2}
\end{gathered}
$$


$\frac{D}{D Z}\left[\frac{\sigma_{3}}{\cos \varepsilon_{3}}\right]=-\left[\frac{\sigma_{3}}{\cos \varepsilon_{3}}\right]\left(\frac{1}{R_{2}} \frac{\partial w_{2}}{\partial Z}+\frac{W}{R_{1}}\right)+\left(\frac{\sigma_{1}}{R_{2} \cos \varepsilon_{3}}\right) \frac{\partial w_{2}}{\partial Z}+\left(\frac{\sigma_{2}}{R_{1}}\right) \frac{\partial w_{2}}{\partial Z} \frac{\partial \varepsilon_{3}}{\partial \varepsilon_{1}}-\rho g \frac{\partial w_{2}}{\partial Z} \cos \varepsilon_{2}$

or

$$
\begin{aligned}
& \frac{\partial}{\partial Z}\left[\frac{\sigma_{3}}{\cos \varepsilon_{3}}\right]=-\frac{\partial}{\partial \varepsilon_{1}}\left[\frac{\sigma_{3}}{\cos \varepsilon_{3}}\right] \frac{\partial \psi_{1}}{\partial X} \frac{D X}{D Z}-\frac{\partial}{\partial X}\left[\frac{\sigma_{3}}{\cos \varepsilon_{3}}\right] \frac{D X}{D Z}-\left[\frac{\sigma_{3}}{\cos \varepsilon_{3}}\right]\left(\frac{1}{R_{2}} \frac{\partial w_{2}}{\partial Z}+\frac{W}{R_{1}}\right) \\
& +\left(\frac{\sigma_{1}}{R_{2} \cos \varepsilon_{3}}\right) \frac{\partial w_{2}}{\partial Z}-\left(\frac{\sigma_{2}}{R_{1}}\right) \frac{\partial w_{2}}{\partial Z} \frac{\partial \varepsilon_{3}}{\partial \varepsilon_{1}}-\rho g \frac{\partial w_{2}}{\partial Z} \cos \varepsilon_{2}
\end{aligned}
$$

A force balance is also conducted on the flow channel, using a Janssen approach(Janssen, 1895).

A vertical force balance over an incremental element $\mathrm{dZ}$ gives:

$$
\begin{aligned}
& \frac{d \sigma_{f v}}{d Z}=\left\{\frac{\tau_{f} P_{f}+\tau_{f w} P_{f w}}{A_{f}}\right\}-\rho g \\
& \tau_{f}=K_{f} \mu \sigma_{f h} \\
& \tau_{f w}=\mu_{w} \sigma_{f h w}
\end{aligned}
$$

$\mathrm{P}_{\mathrm{f}}$ is the internal flow channel half perimeter $\mathrm{m}$

$\mathrm{P}_{\mathrm{fw}}$ is the flow channel wall perimeter $\mathrm{m}$

$\mathrm{A}_{\mathrm{f}}$ is the flow channel half area $\mathrm{m}^{2}$

$\sigma_{\mathrm{fv}}$ is the flow channel vertical stress $\mathrm{Pa}$

$\sigma_{\text {fh }}$ is the flow channel horizontal, normal stress $\mathrm{Pa}$

$\tau_{\mathrm{f}}$ is the flow channel shear stress $\mathrm{Pa}$ (assumed to act upwards)

$\tau_{\mathrm{fw}}$ is the flow channel wall shear stress $\mathrm{Pa}$

$\mu$ is the internal coefficient of friction

$\mu_{\mathrm{w}}$ is the coefficient of wall friction

The incremental element is curved to follow the flow channel border. Areas are projected area onto the horizontal plane.

$$
\begin{aligned}
& A_{f}=\frac{1}{2} r_{2}^{2}\left(\theta_{o}-\sin \theta_{o}\right)+\frac{1}{2} r_{1}^{2}\left(\left(\frac{\pi}{2}-\theta_{o}\right)-\sin \left(\frac{\pi}{2}-\theta_{o}\right)\right) \\
& P_{f}=r_{1}\left(\frac{\pi}{2}-\theta_{o}\right) \\
& P_{f w}=r_{2} \theta_{o}
\end{aligned}
$$

A horizontal stress is given by:

$$
\sigma_{f h}=K_{f} \sigma_{f v}
$$


$\mathrm{K}_{\mathrm{f}}$ is the flow channel stress ratio: horizontal stress/vertical stress

\section{Boundary Conditions}

The changes in geometry require modifications in some of the boundary conditions.

Equation 19 requires a boundary condition in $Z$, referred to reference surface coordinate $Z_{0}$ :

$$
Z=Z_{o}: \sigma_{f v}=\sigma_{f v o}
$$

$\sigma_{\mathrm{fh}}$ interacts with the bulk to form a boundary condition in $\sigma_{2}$, equation 16 .

$$
\varepsilon_{1}=\pi-\theta: \sigma_{2}=\sigma_{f h}
$$

noting that for $\varepsilon_{1}>=\pi-\theta$ the conditions in the flow channel apply, and for $\varepsilon_{1}<\pi-\theta$, stresses are calculated using equations 16-19.

Other boundary conditions include(Matchett et al 2015, 2016):

Fort equation 18

$$
\begin{aligned}
& Z=Z_{o}: \sigma_{3}=\sigma_{3 o} \\
& X=X_{\max }: \frac{\partial \sigma_{3}}{\partial X}=\gamma_{1} \sigma_{3}
\end{aligned}
$$

And equation 17

$$
\begin{aligned}
& X=0: \frac{\partial \sigma_{1}}{\partial X}=0 \\
& X=0: \frac{\partial \sigma_{2}}{\partial X}=0 \\
& X=0: \sigma_{1}=\sigma_{2}
\end{aligned}
$$

\section{Integration of the force balance equations}

The force balance equations were integrated using a finite difference methodology, in a modified and improved qb64 program. Attention was paid to the stability of the numerical algorithms. Specifically, either forward or backward differences were used in integration of equation 18, dependent upon the sign and value of the coefficients of $\sigma_{3}$, to ensure all coefficients of $\sigma_{3}$ were positive.

This gave a fast and reliable program over a wide range of input parameters.

\section{Comparison of the two region model with the Eurocode 1 calculation}

A model simulation is compared to Eurocode 1 calculations in Figure 11. The model is able to reproduce the stress in the flow channel, but the bulk stresses are too small. For the silo conditions in Figure 11, this was the "best fit" that could be found. The bulk stresses could be 
increased by making $\beta_{\mathrm{c}}>0$, but this creates conceptual, mathematical and numerical problems and could not be accepted as a possible solution.

The internal stress structure is indicated in Figure 12, where the principal stresses are plotted along the x-axis. $\sigma_{3}$ is much greater than the other two principal stresses for most of the axis. This is reflected in the Conical Yield Function (CYF). In order to aid representation a Conical Yield Function Index is defined:

$$
\begin{aligned}
& \mathrm{CYFI}=1 \text { if } \mathrm{CYF}>=0 \\
& \mathrm{CYFI}=-1 \text { if } \mathrm{CYF}<0
\end{aligned}
$$

Figure 13 shows CYFI in $\mathrm{X}-\varepsilon_{1}$ space. Clearly, most of the bulk space is above the yield locus. Values of CYFI between +1 and -1 are an artifact of the surface plotting function in Excel, which was designed to model smooth surfaces.

Therefore, a system must be found in which the stresses transmitted to the bulk solids across the flow channel internal wall are increased. The flow channel cannot generate sufficient horizontal stress for the high, vertical stress within the central region of the bulk solids.

A transition zone is required. This is equivalent to the high stress zone in Eurocode 1(2006). It would be cynical to introduce an intermediate zone between the flow channel and the bulk solids just because there is one in Eurocode 1. However, initial modelling with a two region model has provided sound practical and conceptual reasons for a transition zone:

1. A transition zone provides a "buffer" between the relatively low stress regime of the flow channel and the high stresses within the central bulk region

2. The transition zone also provides a break between the dynamic flow channel and the static, steady state principal stress cap zone

3. The transition zone should allow sufficiently high values if $\sigma_{2}$ and $\sigma_{1}$ such that a greater proportion of the silo remains within the CYF at any depth

4. Horizontal force balances can be satisfied

\section{The three zone model for a wall flow channel}

The principal stress cap model can be modified to introduce a transition zone between the flow channel and the bulk solid principal stress cap zone.

This is conveniently done by introducing an inner circular arc, inside that marked out by angle $\theta_{\mathrm{o}}$. This has arc angle $\eta$ and half-chord length $\mathrm{l}_{\mathrm{f}}$ - Figure 14. Thus, the flow channel is now bounded by angle $\eta$, and the transition zone by angle $\theta_{0}$. 
A subscript convention has been employed whereby the first letter of the subscript denotes the zone:

subscript f-flow channel

subscript $\mathrm{t}$ - transition zone

The second letter refers to either:

$\mathrm{v}$ - vertical

h - horizontal

A subscript w denotes the silo wall, as opposed to an internal surface

Force balances on the flow channel are controlled by equations 19 and 20, but geometrical parameters are modified and the flow channel radius is now referred to as $r_{3}$ :

$$
\begin{aligned}
& A_{f}=\frac{1}{2} r_{2}^{2}(\eta-\sin \eta)+\frac{1}{2} r_{3}^{2}\left(\left(\frac{\pi}{2}-\eta\right)-\sin \left(\frac{\pi}{2}-\eta\right)\right) \\
& P_{f}=r_{3}\left(\frac{\pi}{2}-\eta\right) \\
& P_{f w}=r_{2} \eta \\
& r_{3}=r_{2} \tan \eta \\
& l_{f}=r_{2} \sin \eta=r_{3} \cos \eta
\end{aligned}
$$

The geometry of the transition zone is now described as:

$$
\begin{aligned}
& P_{t}=r_{1}\left(\frac{\pi}{2}-\theta_{o}\right) \\
& P_{t w}=r_{2}\left(\theta_{o}-\eta\right) \\
& A_{t}=\frac{1}{2} r_{2}^{2}\left(\theta_{o}-\sin \theta_{o}\right)+\frac{1}{2} r_{3}^{2}\left(\left(\frac{\pi}{2}-\theta_{o}\right)-\sin \left(\frac{\pi}{2}-\theta_{o}\right)\right)-A_{f}
\end{aligned}
$$

Where:

$\mathrm{Pt}$ is the internal half-perimeter between the transition zone and the bulk $\mathrm{m}$ $\mathrm{P}_{\mathrm{tw}}$ is the wall perimeter of the transition zone $\mathrm{m}$ $A_{t}$ is the cross section area of the transition zone $\mathrm{m}^{2}$

Figure 15 shows the stresses acting upon the transition zone element $\mathrm{dZ}$. It is assumed that the interface between the transition zone and the bulk is a plane of principal stress, such that the horizontal principal stress is transmitted across the interface into the bulk: $\sigma_{2 b}$ in Figure 15. This acts as a boundary condition - as in equation 22. There are no shear forces on this interface. 
The assumption of principal stresses acting on the border of the transition zone is made for a number of reasons:

- It is necesssary for implementation of the principal stress cap model

- The borders between the zones are arbitrary - Eurocode 1 suggests calculations for at least 3 flow channel sizes - Eurocode 1(2016), page 53

- Therefore, it is reasonable to select a location of boundary which is a principal stress plane, or a close approximation

The vertical force balance is given by:

$$
\frac{\partial \sigma_{t v}}{\partial Z}=\frac{\left(-\tau_{f} P_{f}+\tau_{t w} P_{t w}\right)}{A_{t}}-\rho g
$$

The horizontal force balance is:

$$
\sigma_{t h w}=\frac{\left(\sigma_{t h} l_{o}-\sigma_{f h} l_{f}\right)}{\left(l_{o}-l_{f}\right)}
$$

$\sigma_{\mathrm{tv}}$ is the vertical stress in the transition zone $\mathrm{Pa}$

$\sigma_{\mathrm{th}}$ is the horizontal stress in the transition zone $\mathrm{Pa}$

$\sigma_{\text {thw }}$ is the horizontal stress at the wall in the transition zone $\mathrm{Pa}$

$\tau_{\mathrm{tw}}$ is the wall shear stress in the transition zone $\mathrm{Pa}$

and

$$
\begin{aligned}
& \sigma_{t h}=K_{t} \sigma_{t v} \\
& \tau_{t w}=\mu_{t w} \sigma_{t h} \\
& \sigma_{2 b}=K_{t b} \sigma_{t h}
\end{aligned}
$$

$\mathrm{K}_{\mathrm{t}}$ is the averaged stress ratio constant for the vertical force balance

$\mathrm{K}_{\mathrm{tb}}$ is the averaged force ratio for the horizontal the force balance

The $\mathrm{K}$ and $\mu$ values are not necessarily limiting values.

A negative value of $\mu$ indicates that the force acts in the opposite direction to that shown in Figure 15. It is primarily a directional device. The direction of $\tau_{\mathrm{f}}$ shown in Figure 15 is equivalent to an upwards, vertical shear stress acting upon the material in the flow channel. A reversal of direction implies rotation within the flow channel, which is completely compatible with the assumption of frictional flow.

The transition/bulk boundary is curved to follow the line of principal stress as in equation 11. The wall and flow channel boundaries are horizontally planar. 


\section{Wall normal stress behaviour of the three-zone model}

Typical wall normal stress behaviours are shown in Figure 16. The flow channel and transition zone are clearly present.

The wall normal stress in the bulk zone is not constant(unlike Eurocode 1). The stress drops in the vicinity of the transition zone boundary. It then rises with $\beta_{\mathrm{w}}>0$, equivalent to passive stress with a convex principal stress cap in the bulk, or passes through a local maximum and then falls for $\beta_{\mathrm{w}}<0$, equivalent to an active stress with a concave principal stress cap in the bulk, as $\varepsilon_{1}$ decreases - see Matchett et al(2016).

\section{Comparison of the three-zone model with stress predictions for Chen et al(2007) experimental data}

Chen et al(2007) conducted extensive tests on a full-size circular silo. Figure 16 of their paper shows extensive wall normal stress data at incipient flow. This has been calculated from a large array of strain gauges mounted on the silo wall. The conditions and materials used are shown in Table 1.

The data of Chen et al(2007) is presented as percent change from the steady stress values. In order to convert to actual stresses, the data were scaled using the stresses calculated from the Eurocode 1 calculation procedure. The range of peak data were scaled by the range between flow channel stress and high stress/transition zone stress of Eurocode 1.

The Chen et al(2007) data show complex patterns. In general, there is a low, minimum stress at the centre of the flow channel $\left(\varepsilon_{1}=180^{\circ}\right)$. The stress then increases rapidly with azimuthal angle(decrease in $\varepsilon_{1}$ ) to a peak corresponding to the high stress/transition zone. It then changes through a series of oscillation. Rings A, C and D have a tendency to a general decrease in stress around the circumference, whereas B shows a tendency to increase. It is suggested that the reader study Figure 16 in the original paper(Chen et al, 2007) to appreciate the details and complexities of the data. The lines presented in this paper are quantitative trend lines taken from the original.

The data have been fitted to the model "by eye", and three simulations were performed. The three simulations are shown in Figures 17. Values of parameters are given in Table 2.

A statistical approach has not been used for several reasons:

- a great deal of effort would be required for a questionable or insignificant gain 
- there are a number of criteria that could be used to generate a fit: overall data comparison: an optimisation of some or just one of the three zones e.g best fit the bulk zone only

- these data were fitted to the model by prioritising the prediction of flow channel and transition stresses in S1 and S2. The bulk stress region was the adjusted without changing the other values, by adjusting wall angle $\beta_{\mathrm{w}}$ and wall gradient factor $\gamma_{1}$

- The limiting value of wall friction was used for the channel: $\mu_{\mathrm{fw}}$ 
Table 1 The conditions of Chen et al(2007) experimental data

\begin{tabular}{|c|c|}
\hline Silo properties & \\
\hline Silo diameter & $4.2 \mathrm{~m}$ \\
\hline Barrel section height & $9.5 \mathrm{~m}$ \\
\hline Measurement ring depth: & \\
\hline A & $3.5 \mathrm{~m}$ \\
\hline B & $5 \mathrm{~m}$ \\
\hline $\mathrm{C}$ & $6.5 \mathrm{~m}$ \\
\hline $\mathrm{D}$ & $8 m$ \\
\hline \multicolumn{2}{|l|}{ Bulk Material Properties } \\
\hline \multicolumn{2}{|l|}{ Material used: iron ore pellets } \\
\hline bulk density & $2300 \mathrm{~kg} / \mathrm{m}^{3}$ \\
\hline angle of wall friction & $26.2^{\circ}$ \\
\hline angle of internal friction & $52^{\circ}$ \\
\hline Limiting coefficient of wall friction & 0.5 \\
\hline Limiting coefficient of internal friction & 0.91 \\
\hline Limiting principal stress ratio(Mohr Circle) & 8.2 \\
\hline Further details in Rotter et al(1995) & \\
\hline
\end{tabular}

Table 2 Values of parameters used in simulations of the Chen et al(2007) data

\begin{tabular}{|c|c|c|c|c|c|c|c|c|c|c|c|}
\hline $\begin{array}{c}\text { Simulation } \\
\text { Number }\end{array}$ & $\begin{array}{c}\boldsymbol{\theta}_{\mathbf{o}} \\
\mathbf{d e g}\end{array}$ & $\begin{array}{c}\eta \\
\mathbf{d e g}\end{array}$ & $\boldsymbol{\mu}$ & $\boldsymbol{\mu}_{\mathbf{f w}}$ & $\boldsymbol{\mu}_{\mathbf{t w}}$ & $\mathbf{K}_{\mathbf{f}}$ & $\mathbf{K}_{\mathbf{t}}$ & $\mathbf{K}_{\mathbf{t b}}$ & $\boldsymbol{\beta}_{\mathbf{w}}$ & $\gamma_{\mathbf{1}}$ & \\
$\mathbf{d e g}^{-1}$ & $\mathbf{m}^{-1}$ & & & & & & & & \\
\hline $\mathbf{S 1}$ & 32.7 & 20.8 & -0.11 & 0.5 & 0.25 & 3 & 0.325 & 0.175 & -4 & 0.1 & \\
\hline $\mathbf{S 2}$ & 32.7 & 20.8 & -0.11 & 0.5 & 0.25 & 3 & 0.325 & 0.175 & 15 & -30 & \\
\hline $\mathbf{S 3}$ & 32.7 & 20.8 & -0.11 & 0.5 & 0.25 & 3 & 0.365 & 0.175 & 17 & -30 & \\
\hline
\end{tabular}

Simulation Number 1(S1): the model outputs were fitted to data for all rings A, B, C and D. The parameters of the flow channel and transition zone were adjusted to give a good prediction of the experimental data. The principal stress cap parameters $\beta_{\mathrm{w}}$ and $\gamma_{1}$ were then 
adjusted to give a fit to the experimental data. This resulted in a value of $\beta_{\mathrm{w}}$ of $-4^{\mathrm{o}}$ giving a concave principal stress cap with an active state of stress.

The value of $\beta_{\mathrm{w}}$ was selected because it is the smallest magnitude negative value that produces bulk zone stresses within the Conical Yield Locus(CYF). Below $-4^{\mathrm{o}}$, areas of the bulk zone which exceed the CYF begin to appear.

This is the best overall simulation of those presented, giving reasonable agreement, both qualitatively and quantitatively over the complete experimental data set of four rings.

Simulation Number 2(S2): the data were fitted to experimental data from Rings A and B only, giving priority to prediction of flow channel and transition zone stresses. A passive stress system was chosen in the bulk zone with $\beta_{\mathrm{w}}>0$ : $\beta_{\mathrm{w}}=15^{\circ} . \beta_{\mathrm{w}}=15^{\circ}$ was chosen as the maximum value with all of the bulk zone remaining within the CYF.

Simulation Number 3(S3): conditions were modified from S2 to fit the whole of the three zones to the experimental data for Rings A and B only. Accuracy of prediction in the flow channel and transition zones was sacrificed for closer correspondence to experimental data in the bulk zone.

\section{Discussion}

There have been several problems in the development of this model. In conceptual terms, the principal stress cap model is explicitly static, but is being used to model an essentially dynamic system of channel flow. The use of a transition zone, as a barrier between the dynamic flow channel zone and the static bulk has had some success in overcoming the problems. The purposes of the transition zone are:

1. to shelter the relatively low stress flow channel from the high stress region around the centre of the silo - see Figure 11

2. to allow high stresses at the transition/bulk interface, independent of the low stresses in the flow zone, thereby keeping stresses within the yield locus

3. to form a transition between the dynamic flow channel and the static bulk

4. to allow a transition from the passive stress state in the flow channel to the active stress state in the bulk

The transition zone contains complex stress systems, ranging from active at one boundary, to passive at the other. It can be argued that it is the most challenging of the three zones to analyse. It might even be said that all the really difficult problems have been lumped into the 
transition zone and ignored, but they are really beyond the scope of this paper and a topic for future work. This is a challenging area for further research.

Lack of experimental data hinders the modelling effort.

There are many issues to be considered when fitting silo models to experimental data, as discussed in Matchett et al(2016). There are major problems in trying to infer internal principal stress orientations from wall stress readings and vice versa. The authors propose that these problems are generic and not confined to the present model. For example, in the Janssen(1895) equation, the Janssen coefficient is given by:

$$
b_{\text {Janssen }}=\frac{4 \mu_{w} K}{d}
$$

$\mathrm{K}$ is the ratio of horizontal to vertical stress

$\mu_{\mathrm{w}}$ is the coefficient of wall friction

$\mathrm{d}$ is the silo diameter $\mathrm{m}$

The Janssen coefficient can be determined from experimental wall stress data, from which the product $\mathrm{K} \mu_{\mathrm{w}}$ can be found. Neither $\mathrm{K}$ nor $\mu_{\mathrm{w}}$ can be found individually without additional information: from the data the internal and wall properties inherent in Janssen cannot be found without further information. For example, the coefficient of wall friction could be measured externally and assumed to be at the limit. Similarly, vertical wall friction could be measured within the silo, as well as normal stress, giving an "in-situ" value of $\mu_{\mathrm{w}}$. However, if one were to question the validity of the Janssen assumption that vertical stress is constant across the horizontal section of the silo(ie internal stress structure), neither of these measurements would provide insights.

In the context of the approach used in this paper, the proposed model is not the only principal stress cap geometry that could be proposed. The present model uses boundary conditions as a basis for interpolation within the cross section of the silo. It is a natural development of previous models(Matchett et al 2015, 2016) and is amenable to mathematical analysis. But it is not unique. As far as the authors are aware, there are no experimental data of internal stress distributions with which to calibrate this, or any other internal, structural model.

$\mathrm{S} 1$ is a reasonable qualitative fit to the data over its full range. The stresses in the flow channel and transition zone are accurately predicted - within +/- $200 \mathrm{~Pa}$. Overall, the 
difference between the model and Chen et al data has a mean value of $581 \mathrm{~Pa}$ with 0.05 confidence limits of $1310 \mathrm{~Pa}$. This is reasonable for data with a maximum value of $24000 \mathrm{~Pa}$. There are discrepancies at the borders between the flow channel/transition zone and transition zone/bulk due to the simplifications of the Janssenian models used, and the location of the borders can have a great effect upon the apparent goodness of fit of the data. For example, if the data points close to the transition zone border are eliminated(these are the points where the difference is greatest) the mean difference between model and data is $-195 \mathrm{~Pa}$ with a 0.05 confidence level of $666 \mathrm{~Pa}$. It is clear that the conditions within the flow and transition zones are simplifications, and that location of borders between zones is arbitrary.

Wall normal stress at the transition/bulk border is relatively low, followed by a general rapid increase in stress in the border region as $\varepsilon_{1}$ decreases, from a relatively low to a maximum bulk zone value. The stress then decreases as $\varepsilon_{1}$ tends to zero. This is in qualitative agreement with the experimental data, except perhaps for Ring B.

The model does not predict the oscillations present in the experimental. Likewise, it could not predict dynamic phenomena, such as shown in Figure 15 of the Chen et al(2007) paper. The oscillations within the bulk zone could be due to a number of causes, for example:

- transmission of stress waves originating within the shear zone, due to flow phenomena and slip.

- discontinuities due to slip and rupture zones within the bulk zone

Unfortunately, the exact causes cannot be determined without further information. This is clearly an aspect of Eurocode 1 that requires further experimental investigation.

S1 has not been optimised, other than "by eye". It could form the starting point of a systematic minimisation of differences. The parameters of S1 apply reasonably well over the full extent of Rings A, B, C and D both qualitatively and quantitatively. The model can scale both azimuthally and with depth, with an active stress state in the bulk zone.

Therefore, the principal stress cap surface model can be applied to systems with a flow channel adjacent to the silo wall and model the bulk solids zone reasonably well.

However, many issues remain:

- Why are data from Ring B somewhat different to the others, with rising/steady bulk zone stresses as $\varepsilon_{1}$ tends to zero.

- The greatest deviations are in the region of the transition/bulk zone interface. Part of this is due to the selection of Janssen(1895)-type models for the flow and transition zones and the choice of the location of the borders. The exclusion of the large 
difference data points from the statistics greatly reduces the magnitude of difference between the model and the data. Models with greater detail are required for these zones and the nature and location of the borders requires further investigation, if indeed, the zones actually exist other than as a theoretical convenience.

- The oscillations inherent in the data are not present in the model. Are they dynamic in nature?

- To what extent does experimental error influence these data?

Clearly, it would be extremely useful to have many more data sets of the extensive nature of Chen et al(2007), to help answer these points.

The stress systems in the present model are very complex, and agreement between model and data in one part of the silo does not imply universal applicability. S2 and S3 have been presented as a case study of potential pitfalls. S2 shows agreement in stresses within the flow channel and transition zone, as S1, but with a passive(convex principal stress cap)) bulk zone. The bulk zone stress for S2 is approximately an upper envelope for the experimental data for Rings A and B only.

S3 is a development of S2 in which accuracy of prediction of flow channel and transition zone wall normal stresses has been sacrificed somewhat in order to provide a better fit between model and data in the bulk zone, again for Rings A and B only.

In the absence of other information, either of these simulations might be considered acceptable.

However, when the simulations are plotted against the experimental data for Rings C and D, it can be seen that the models show poor qualitative and quantitative agreement. In particular, the model shows an increase in wall stress as $\varepsilon_{1}$ decreases towards zero, while the experimental data decrease as $\varepsilon_{1}$ tends to zero. Therefore, S2 and S3 can be used as empirical predictors in the region of Rings A and B, but are not more widely applicable.

A modeller might find reasons for rejecting the data from Rings $C$ and $D$ :

- $\mathrm{C}$ and $\mathrm{D}$ are nearer the surface and might be suffering from surface effects

- The stresses might not be fully developed in the region of $\mathrm{C}$ and $\mathrm{D}$

These arguments would be plausible if it were not for the fact that an active bulk zone(S1) describes the full extent of these data.

Therefore, it may be deduced that large data sets are required to calibrate and test models thoroughly. Comparison with limited data sets will give limited validity and produce only 
empirical relationships valid over a restricted range of conditions. Limited data may suggest misleading models of internal stress structure. Data points which do not agree with a proposed model should not be arbitrarily rejected unless there is evidence to justify such rejection. A corollary of this argument is that extrapolation beyond data limits can be very misleading.

However, even for S1, it is wise to remember to old adage "just because a model can be fitted to experimental that does not necessarily mean that it is "true". We always need more evidence.

Finally, this study of wall flow channels has made the authors appreciate the quality of the analysis inherent in Eurocode 1. The authors of Eurocode 1 have taken a tremendously complex stress situation and developed a design procedure that encapsulates and simplifies into a workable set of procedures.

\section{Conclusions}

A flow channel in the vicinity of the wall of a cylindrical silo, as described in Eurocode 1(2006), has been modelled by a three-zone model, consisting of:

- the flow channel

- the transition zone

- the bulk of the solids

The flow channel and the transition zone were modelled by Janssen-type equations. The bulk solid was modelled by the principal stress cap surface approach.

The paper has shown that the principal stress cap surface approach can be applied to this type of system.

The model was compared and calibrated against experimental data from a full scale silo by Chen et al(2007). The model was able to describe the data both axially and azimuthally reasonably well.

An illustration of the pitfalls of comparing wall stress data with models has been shown in reference to the model and the Chen data.

Large experimental data sets are required to calibrate and test a model. Furthermore, unfortunate data points cannot be arbitrarily rejected.

Improved models of the flow channel and transition zone are needed. 
More experimental data are required to calibrate existing models and help develop new models.

\section{References}

Chen J.F., J.M.Rotter, J.Y.Ooi, Z.Zhong, 2007, Correlation between the flow pattern and wall pressures in a full scale experimental silo. Engineering Structures, 29, 2308-2320

Enstad G., 1975, On the theory of arching in mass flow hoppers, Chem.Eng.Sci. 30, 1273 1283

Eurocode 1, EN 1991-4, 2006. Action on structures - Part 4: Silos and Tanks. Brussels, Belgium.

Janssen H.A., 1895. Versuche uber getreidedruck in silozellen, Zeitschrift verein Deutsche Inginieur, 39,1045-1049

Lapko A., 2010, Pressure of agricultural bulk solids under eccentric discharging of cylindrical concrete silo bin. International Agrophysics, 24, 51-56

Matchett A.J., P.A.Langston, D.McGlinchey. 2015. A model for stresses in a circular silo with an off-centre circular core, using the concept of a principal stress cap: Solutions for a completely filled silo and comparison with DEM data. Chemical Engineering Research and Design 93, 330-348

Matchett A.J., P.A.Langston, D.McGlinchey. 2016. A "principal stress cap" model for stresses in a circular silo with an off-centre circular core: Finite core models, including filled silos, incipient flow and switch stresses. Chemical Engineering Research and Design 106, 263-282

Nedderman R.M. 1992, Statics and Kinematics of Granular Materials, Cambridge University Press, Cambridge, England

Rotter J.M, J.Y.Ooi, I.F.Chen, P.J.Tiley, I.McIntosh, F.R.Bennett. 1995. Flow pattern measurement in full scale silos. British Materials Handling Board Publications, London.

Sielamovicz I., M.Czech, T.A Kowalewski. 2010. Empirical description of flow parameters in eccentric flow inside a silo model, Powder Technology 198, 381-394

Sielamovicz I., M.Czech, T.A Kowalewski. 2011. Empirical analysis of eccentric flow registered by the DPIV technique inside a model silo, Powder Technology 212, 38-56

Sielamovicz I., M.Czech, T.A Kowalewski. 2015. Comparative analysis of empirical descriptions of eccentric flow in silo model by the linear and non-linear regressions, Powder Technology 270, 393-410 


\section{Notation}

The notation used in Matchett et $\mathrm{al}(2015,2016)$ is used in this paper. Additional notation used specifically in this paper is given below:

\begin{tabular}{|c|c|c|}
\hline SYMBOL & DESCRIPTION & UNITS \\
\hline $\mathrm{A}_{\mathrm{f}}$ & flow channel zone cross section area & $\mathrm{m}^{2}$ \\
\hline $\mathrm{A}_{\mathrm{t}}$ & transition zone cross section area & $\mathrm{m}^{2}$ \\
\hline $\mathrm{b}_{\text {Janssen }}$ & Janssen coefficient & $\mathrm{m}^{-1}$ \\
\hline CYFI & Conical Yield Function Index & - \\
\hline $\mathrm{d}$ & silo diameter & $\mathrm{m}$ \\
\hline$\overline{\mathrm{K}_{\mathrm{f}}}$ & flow channel zone stress ratio: horizontal/vertical & - \\
\hline $\mathrm{K}_{\mathrm{t}}$ & transition zone stress ratio: horizontal/vertical & - \\
\hline $\mathrm{K}_{\mathrm{tb}}$ & $\begin{array}{l}\text { transition zone stress ratio: horizontal/vertical, used in horizontal } \\
\text { force balance }\end{array}$ & - \\
\hline 1 & half chord length subtended by angle $\theta$ & $\mathrm{m}$ \\
\hline $1_{\mathrm{o}}$ & half chord length subtended by angle $\theta_{\mathrm{o}}$ : transition zone & $\mathrm{m}$ \\
\hline $\mathrm{l}_{\mathrm{f}}$ & flow channel half chord length & $\mathrm{m}$ \\
\hline$\overline{P_{f}}$ & flow channel half perimeter & $\mathrm{m}$ \\
\hline $\mathrm{P}_{\mathrm{fw}}$ & flow channel wall perimeter & \\
\hline$r_{3}$ & flow channel radius & $\mathrm{m}$ \\
\hline$\theta$ & $\begin{array}{l}\text { angle subtended between the silo centre and a point at the bulk } \\
\text { solid zone/transition zone boundary }\end{array}$ & - \\
\hline$\theta_{\mathrm{o}}$ & $\begin{array}{l}\text { angle subtended between the silo centre and the point where the } \\
\text { bulk solid zone boundary meets the silo wall }\end{array}$ & - \\
\hline$\mu$ & internal coefficient of friction & - \\
\hline$\mu_{\mathrm{w}}$ & coefficient of wall friction & - \\
\hline$\mu_{\mathrm{tw}}$ & transition zone coefficient of wall friction & - \\
\hline$\sigma_{\mathrm{fh}}$ & flow channel horizontal stress & $\mathrm{Pa}$ \\
\hline$\sigma_{\mathrm{fv}}$ & flow channel vertical stress & $\mathrm{Pa}$ \\
\hline
\end{tabular}




\begin{tabular}{|c|l|c|}
\hline$\sigma_{\text {th }}$ & transition zone horizontal stress & $\mathrm{Pa}$ \\
\hline$\sigma_{\mathrm{thw}}$ & transition zone horizontal wall stress & $\mathrm{Pa}$ \\
\hline$\sigma_{\mathrm{tv}}$ & transition zone vertical stress & $\mathrm{Pa}$ \\
\hline$\sigma_{2 \mathrm{~b}}$ & $\begin{array}{l}\text { principal stress } \sigma_{2} \text { transmitted across the transition/bulk interface: } \\
\text { boundary condition for the bulk zone }\end{array}$ & $\mathrm{Pa}$ \\
\hline$\tau_{\mathrm{f}}$ & flow channel internal shear stress & $\mathrm{Pa}$ \\
\hline$\tau_{\mathrm{fw}}$ & flow channel wall shear stress & $\mathrm{Pa}$ \\
\hline & & \\
\hline
\end{tabular}

The notation used in the previous papers (Matchett et al 2015, 2016) is given below:

\begin{tabular}{|c|c|c|}
\hline SYMBOL & DESCRIPTION & UNITS \\
\hline $\mathrm{a}_{1}$ & Relates projected circle radius $\mathrm{R}_{1}$ to $\mathrm{X} \quad R_{1}=a_{1} X+a_{1}{ }^{\prime}$ & - \\
\hline$a_{1}^{\prime}$ & Constant relating $\mathrm{R}_{1}$ to $\mathrm{X}-$ see $\mathrm{a}_{1}$ & $\mathrm{~m}$ \\
\hline$a_{2}$ & Differential of projected circle centre $\quad a_{2}=\partial O_{X} / \partial X$ & - \\
\hline $\mathrm{D}$ & $\begin{array}{l}\mathrm{D} / \mathrm{DX} \text { and } \mathrm{D} / \mathrm{DZ} \text { are differentials along the principal stress paths } \\
\text { for changes in } \mathrm{X} \& \mathrm{Z} \text { respectively }\end{array}$ & \\
\hline $\mathrm{e}_{1}$ & Angle used in the calculation of $\mathrm{R}_{2}$ & $\mathrm{rad}$ \\
\hline $\mathrm{E}$ & $\begin{array}{l}\text { Factor relating rotation in the horizontal plane to rotation on the } \lambda \text { - } \\
\text { plane }\end{array}$ & - \\
\hline $\mathrm{h}$ & Inner circle offset & $\mathrm{m}$ \\
\hline & & - \\
\hline $\mathrm{k}$ & Ratio of wall vertical to normal stress: Janssen model & - \\
\hline $\mathrm{K}_{\mathrm{w}}$ & Ratio of $\sigma_{1} / \sigma_{3}$ at the wall & - \\
\hline $\mathrm{M}$ & Conical Yield Function parameter & - \\
\hline $\mathrm{r}_{1}$ & Inner circle radius & $\mathrm{m}$ \\
\hline $\mathrm{r}_{2}$ & Outer circle (silo) radius & $\mathrm{m}$ \\
\hline $\mathrm{R}_{1}$ & Radius of projected horizontal circle of principal stress path & $\mathrm{m}$ \\
\hline $\mathrm{R}_{2}$ & Radius of principal stress cap at a general point & $\mathrm{m}$ \\
\hline $\mathrm{R}_{20}$ & Value of $\mathrm{R}_{2}$ at $\varepsilon_{1}=0$ & $\mathrm{~m}$ \\
\hline $\mathrm{R}_{2 \pi}$ & Value of $\mathrm{R}_{2}$ at $\varepsilon_{1}=\pi$ & $\mathrm{m}$ \\
\hline
\end{tabular}




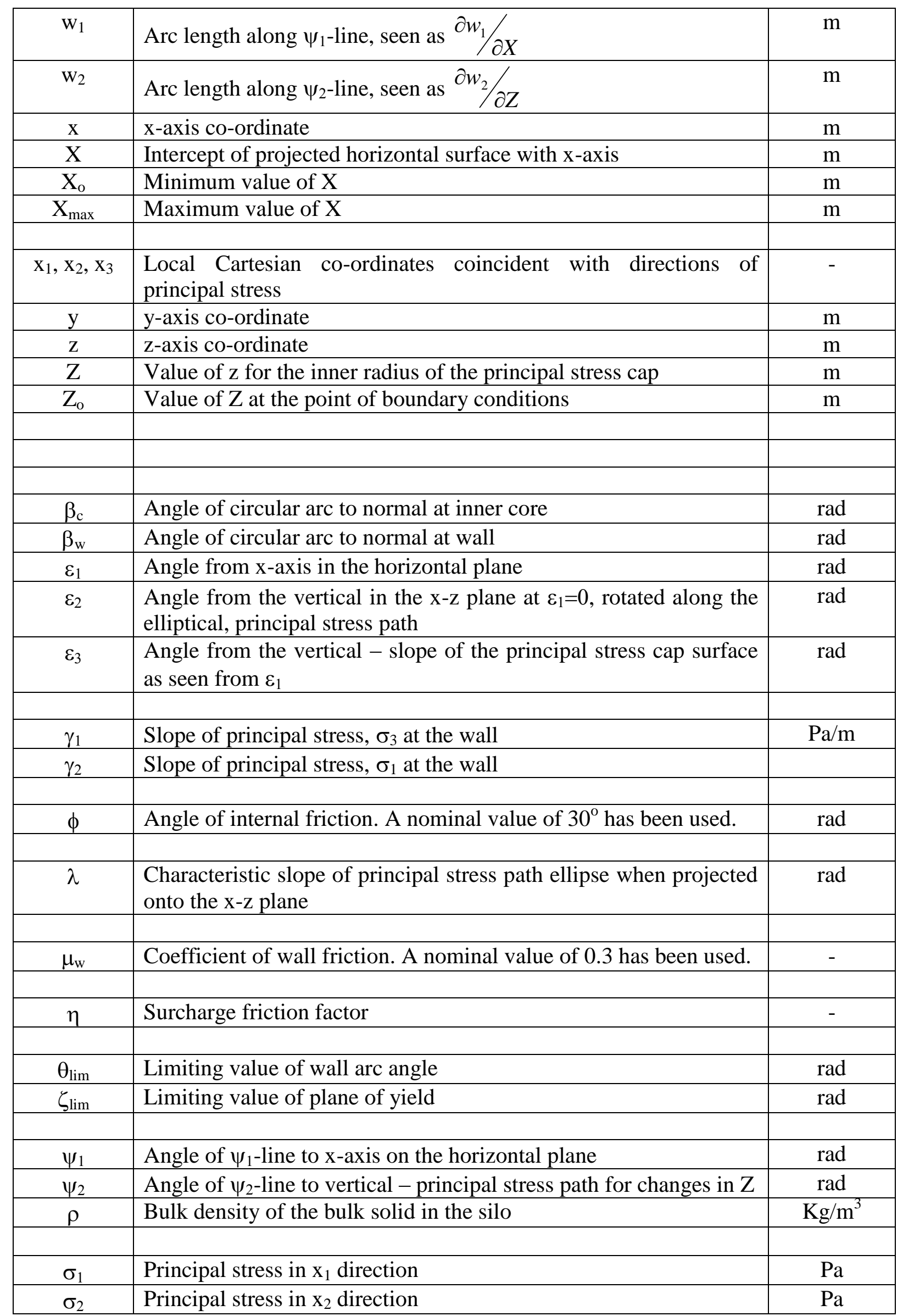




\begin{tabular}{|c|l|c|}
\hline$\sigma_{3}$ & Principal stress in $\mathrm{x}_{3}$ direction & $\mathrm{Pa}$ \\
\hline & & \\
\hline & & \\
\hline & & \\
\hline & & \\
\hline
\end{tabular}


A continuum model of stresses in a vertical silo with a flow channel in the vicinity of the wall, using the principal stress cap surface approach for the bulk solids

\section{A.J.Matchett, University of Teesside(retired) ; P.A..Langston, Faculty of Engineering, University of Nottingham; D.McGlinchey, School of Engineering, Glasgow Caledonian University}

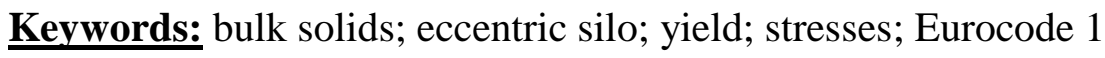

\section{Summary}

Eurocode 1(2006) gives design equations for eccentric stresses in silos, including flow channels adjacent to the wall. This has been modelled using the approach of Matchett et al(2015, 2016).

A three zone model was developed, consisting of:

- the flow channel

- the transition zone

- the bulk of the solids

The flow channel and the transition zone were modelled by Janssen-type equations. The bulk was modelled by the principal stress cap approach.

The transition zone is a complex region and has several purposes:

1. to shelter the low stress flow channel from the high stresses around

2. to allow high principal stresses at the transition/bulk interface, within the yield locus

3. to form a transition between the dynamic flow channel and the static bulk

4. to allow transition from passive stress in the flow channel to active stress in the bulk

The model was calibrated against the data of Chen et al(2007) for a full-scale silo, and described the data reasonably well, scaling axially and azimuthally.

Large experimental data sets are required to calibrate a model. Unfortunate data points cannot be arbitrarily rejected.

Further extensive, experimental data are needed to calibrate models. 


\section{Introduction}

Matchett, Langston and McGlinchey $(2015,2016)$ describe how eccentric stresses in vertical, cylindrical silos were modelled. They used a "principal stress cap surface" approach, in which the geometry of the principal stress cap and principal stress paths in 3-dimensional space were assumed "a priori" - see Figure 1.

Seen from above, the principal stresses in the azimuthal direction describe circular stress path in the horizontal plane.

In a symmetrical system, the circle would be centred about the centre of the silo, of radius $r_{2}$, creating a series of concentric circles. In an asymmetrical system, the centre of the core, radius $r_{1}$, was displaced a distance $h$, causing the circular stress paths to be compacted in the direction of displacement and expanded in the opposite direction. The centre of the core is now the centre of stress, and a Cartesian axis was defined at this point, with $\mathrm{x}$ in the direction of displacement and z vertically. The centres of successive, azimuthal principal stress circles were also displaced, and a given circle can be characterised by the value $\mathrm{X}$ (upper case $\mathrm{x}$ ), its value at the point where it crosses the $\mathrm{x}$ axis.

The vertical height was characterised by parameter $\mathrm{Z}$ (upper case $\mathrm{z}$ ). This is the value of $\mathrm{z}$ at a characteristic point on the principal cap surface - in this case the value of $\mathrm{z}$ at the core. The principal stress cap surface describes a circular arc in the $\mathrm{x}-\mathrm{z}$ plane, along the $\mathrm{x}$-axis, with characteristic radii $R_{20}$ in the positive sense of $x$ and $R_{2 \pi}$ in the negative direction of $x$. $A$ given circle subtends an angle $\varepsilon_{2}$ to the vertical plane. $\varepsilon_{2}$ is constant around a line of constant $\mathrm{X}$. Thus, an X-line describes the path of principal stress $\sigma_{2}$ and is a circular projection onto the horizontal plane of an elliptical path in 3 dimensions.

On the surface of the principal stress cap and normal to the X-lines, are the "radial" stress paths of stress $\sigma_{1}$, given by the $\psi_{1}$-lines. A projection onto the horizontal plane is shown in Figure 2.

The principal stress $\sigma_{3}$ is normal to the stress cap surface - Figure 1, lower section.Its path in space is described by $\psi_{2}$ lines, normal to the principal stress cap surface.

A given $\mathrm{X}$-line is at an angle of $\lambda$ to the vertical, when seen from the $\mathrm{y}$-axis. At a given point $\mathrm{P}$, at rotation angle $\varepsilon_{1}$ is inclined at angle $\varepsilon_{3}$.

All relevant parameters to describe the surface can be deduced from the preceding assumptions: Matchett et al $(2015,2016)$

Genuine 3-dimensional force balances were performed in principal stress space, based upon an incremental element bounded by $X, \psi_{1}$ and $\psi_{2}$ lines, to determine stress distributions 
within the silo. This gives orthogonal equations in $\sigma_{1}, \sigma_{2}$ and $\sigma_{3}$ : see Figure 1 , lower section for local principal stress axes.

The methodology is a true 3-d development of the concept of the principal stress cap first proposed by Enstad: Enstad(1975), Nedderman(1992). It carries forward many of the advantages and limitations of the Enstad approach:

- The models are steady-state. They can only model static systems or systems at incipient flow

- The bulk materials are modelled as rigid-plastic solids: there are no elastic components

- The model applies to the central, axial section of a silo (preferably a slender silo), in which the stresses are fully developed, as opposed to the surface where stresses are affected by the surface interface and any surcharge, or the base where the geometry of the silo bottom affects stress patterns

- The models are relatively simple, both conceptually and numerically, when compared to DEM, FEM and other true 3-d models of bulk solids in silos

- The models predict stress distributions from assumed principal stress orientations. The numerical algorithms are relatively simple and quick, and model outputs can be obtained in tens of minutes, compared to days for some, more sophisticated algorithms

- Post-calculation the validity of the outputs must be tested by some means: Conical Yield Function (CYF) and Wall Yield Function (WYF) were used to assess the validity of model outputs: Matchett et al(2016)

- The models give extensive 3-dimensional data for stress distributions within silos, as well as wall stress data

The following findings were inferred from the simulation data:

1. Completely filled silos, (Matchett et al, 2015) and silos with finite cores (Matchett et al, 2016) were modelled

2. There are at least two types of stress eccentricity in silos: surcharge or surface eccentricity, and inherent eccentricity: Matchett et al(2015)

3. Surcharge eccentricity is due to an uneven distribution of surcharge material on top of an underlying, uniform stress pattern. Eccentricities quickly decay with depth, as per the Janssen model( Jannsen, 1895) 
4. Inherent eccentricity is due to an underlying, off-centre centre-of-stress. Stress eccentricities increase with depth

5. The models were able to predict wall stresses corresponding to a DEM simulation: Matchett et al(2015)

6. A range of internal stress parameters within the model were able to give reasonable approximations to the DEM wall stress output. There were no indications of a unique relationship between internal structure and wall stress distribution, and a range of parameters give reasonable approximations to external data.

7. Large variations in wall normal stress were found at high eccentricities and large wall angles. However, such systems were often found to be non-viable as they exceeded the Yield Loci

8. Expected features of silo behaviour were modelled, including: passive(convex stress cap) systems; active(concave stress cap) systems; incipient flow and switch stress: Matchett et al(2016)

9. Two recurrent themes in the papers were: the need for experimental data for internal stress distributions in silos; the inability to predict internal stress distributions from wall stress measurements

The systems modelled consisted of eccentric cores which did not extend beyond the limits of the silo wall.

Many workers have studied systems where there is a flow channel that touches the silo wall, as shown in Eurocode 1(2006) - Section 5.2.4.3, page 52, Figure 5.5. Other workers have studied systems where the flow channel is in the region of the silo wall, including Sielamovicz et el(2010, 2011, 2015), Chen et al(2007) and Lapko(2010).

This paper seeks to extend the 3-d, principal stress cap technique to cover situations of wall discharge approximating to the Eurocode 1 analysis.

\section{The Eurocode 1 silo design algorithm for a flow channel in the vicinity of the silo wall}

The Eurocode 1 design standard for silos presents a series of equations for the calculation of wall normal stresses for the purposes of structural design of the silo.

The code proposes 3 zones:

- A flowing zone, in which the material is in motion to discharge down the silo

- A static zone far(relatively) from the flowing zone, where the solids remain static

- A high stress zone, between the flow channel zone and the bulk zone 
$\theta_{c}$ is the half-angle subtended between the edge of the flow zone, the silo centre, and the centre of the flow zone. The high stress zone is also assumed to be of angle $\theta_{c}$ beyond the flow channel. The remainder of the silo is assumed to be in the static zone. The code presents equations to calculate wall normal and vertical stresses in each zone. A typical calculation is shown in Figure 3 for the material used in example calculations in previous papers: Matchett et $\mathrm{al}(2015,2016)$. The static bulk material has an intermediate magnitude of stress, the high stress zone is the largest stress, and the flow zone has a relatively low stress. This analysis is very stark compared with the experimental data of Chen et al(2007), but the existence of the high stress zone has been shown by many workers, for example Chen et al(2007) and Lapko(2010).

\section{A two-zone model for a flow channel in the vicinity of the silo wall}

An initial consideration of the situation of a wall flow channel suggests that there are two zones, Figure 4, Sielamovicz et al(2010, 2011), Eurocode 1(2006), Figure 5.5a:

- The flow channel, adjacent to the wall

- The remainder of the silo, containing bulk solids

For the purposes of this modelling exercise, it will be assumed that a region of fully developed stress and steady state flow exists between the surface and the bottom of the silo. This is the region that will be modelled. An equivalent approach was taken in the previous papers(Matchett et al, 2015, 2016).

Consider a horizontal slice through the region of fully developed stress - Figure 5. The silo has radius $r_{2}$ and centre at $\mathrm{O}_{2}$. The silo wall is cut by the flow channel, which is of circular cross-section and radius $r_{1}$. The flow channel section is assumed to cut the silo wall at rightangles. The silo wall is a projected path of circumferential(azimuthal) principal stress, $\sigma_{2}$. Hence, the flow channel wall is a projected path of principal stress $\sigma_{1}$. The point of intersection projects a half-angle $\theta_{\mathrm{o}}$ at the silo centre, and has half-chord length $1_{\mathrm{o}}$. The normality of the silo and flow channel is contrary to Eurocode 1, where the intersection angle is determined by a minimum flow area basis. However, normality is essential in order to implement the principal stress cap model to the bulk zone.

A reference set of Cartesian axes is placed $(x, y, z)$ is placed at the apex of the flow channel border, along the line connecting the two circle centres, with $\mathrm{x}$ and $\mathrm{y}$ in the horizontal plane and $\mathrm{z}$ vertically out of the plane. 
As in the previous models(Matchett et al 2015, 2016), it is assumed that the azimuthal principal stress, $\sigma_{2}$, projects a circular path onto the horizontal plane. A generalised path is shown in Figure 6. The circle has an intercept X(upper case) with the reference X-axis and a radius $\mathrm{R}_{1}$. The general stress path circle cuts the flow channel border normally. The circle has a centre $\mathrm{O}$. The half-angle between the circle centre and the point of intersection with the flow channel wall is $\theta$, with half-chord length 1 .

A general point $\mathrm{P}$ - Figure 6 , can be identified by specifying the circle on which it sits, through its intersection $X$, and the subtended angle between the arc and circle centre $O, \varepsilon_{1}$. The third co-ordinate is provided by $\mathrm{Z}$, the intersection of the characteristic location of the principal stress cap with the z-axis: Matchett et al(2015, 2016).

Within the silo, the $\mathrm{X}$-lines cut the $\mathrm{x}$-axis at various values of $\mathrm{x}$, to give the $\mathrm{X}$ value of each projected circle. These plot the course of azimuthal stress $\sigma_{2}$. The X-lines are crossed by $\psi_{1}$-lines, which mark the paths of principal stress $\sigma_{1}$ - Figure 7. Each $\psi_{1}$ - line has a unique, identifying value marked by the value of angle $\psi_{1}$ at the flow channel border wall of $\psi_{10}$. The flow channel border is therefore, also a $\psi_{1}$-line and marks the limit of the principal stress cap system.

The stress and co-ordinate system is a direct development of the models presented in the previous papers(Matchett et al 2015, 2016), with geometric modifications. The equations from Matchett et al(2015) can be directly transposed to the present system, taking account of geometrical differences. Therefore, this model will be presented in outline only, with emphasis on those changes from the original model. The remainder of the parameters are as in the original model and original notation is included in this paper for convenience.

The reader is referred to Matchett et al(2015) for details.

\section{$\mathrm{X}$-line geometry}

The silo wall, radius $r_{2}$ and the flow channel wall, radius $r_{1}$ cross normally. It can be shown that:

$$
\begin{aligned}
& \sin \theta_{o}=\frac{l_{o}}{r_{2}} \\
& r_{1}=r_{2} \tan \theta_{o}
\end{aligned}
$$

And for a general X-circle with subtended half-angle $\theta$ :

$$
\begin{aligned}
& l=R_{1} \sin \theta=r_{1} \cos \theta \\
& R_{1}=r_{1} \cot \theta
\end{aligned}
$$


and $\theta$ is related to co-ordinate system variable $\varepsilon_{1}$ or $\psi_{1}$ by:

$$
\varepsilon_{1}=\pi-\theta
$$

The value of $\mathrm{X}$ is given by:

$$
X=R_{1}(1+\cos \theta)-r_{1}(1-\sin \theta)
$$

The co-ordinate of the centre of circle $\mathrm{X}, \mathrm{O}$ :

$$
O=R_{1} \cos \theta-r_{1}(1-\sin \theta)
$$

At the silo wall:

$$
\begin{aligned}
& R_{1}=r_{2}: X=X_{\max }: \theta=\theta_{o} \\
& X_{\text {max }}=r_{2}(1+\cos \theta)-r_{1}(1-\sin \theta)
\end{aligned}
$$

The path of a given $\psi_{1}$-line is described by two parameters: $a_{1}$ and $a_{2}$ :

$$
\begin{aligned}
& a_{1}=\frac{\partial R_{1}}{\partial X} \\
& a_{2}=\frac{\partial O}{\partial X}
\end{aligned}
$$

such that:

$$
\begin{aligned}
& \frac{\partial w_{1}}{\partial X}=a_{1}+a_{2} \cos \psi_{1}=a_{1}+a_{2} \cos \varepsilon_{1} \\
& \frac{\partial \psi_{1}}{\partial X}=\frac{a_{2} \sin \psi_{1}}{R_{1}}=\frac{a_{2} \sin \varepsilon_{1}}{R_{1}}
\end{aligned}
$$

hence:

$$
a_{1}=\frac{\partial R_{1}}{\partial X}=-r_{1}^{2} \operatorname{cosec}{ }^{2} \theta \frac{\partial \theta}{\partial X}
$$

and

$$
\begin{aligned}
& a_{2}=\frac{\partial O}{\partial X}=\left\{-R_{1} \sin \theta+\cos \theta \frac{\partial R_{1}}{\partial \theta}+r_{1} \cos \theta\right\} \frac{\partial \theta}{\partial X} \\
& \frac{\partial X}{\partial \theta}=(1+\cos \theta) \frac{\partial R_{1}}{\partial \theta}-R_{1} \sin \theta+r_{1} \cos \theta \\
& \frac{\partial R_{1}}{\partial \theta}=-r_{1} \operatorname{cosec}{ }^{2} \theta
\end{aligned}
$$

The previous models had $\mathrm{a}_{1}$ and $\mathrm{a}_{2}$ as constants. In this version, $\mathrm{a}_{1}$ and $\mathrm{a}_{2}$ are variables and functions of $\theta$ and hence $X$.

The surface geometry of the principal stress cap 
Figure 8 shows a represenation of the principal stress cap surface including a plan view and view from the $y$-axis. Seen from the $y$-axis, the total surface is represented by ABC. AB is a circular arc along and above the $\mathrm{x}$-axis. $\mathrm{BC}$ is the $\lambda$-line at the silo wall. ADE is the portion of the stress cap within the circle $\mathrm{X}$, where DE is the corresponding $\lambda$-line.

Reference co-ordinate $\mathrm{Z}$ is the height of the apex of the principal stress cap/flow channel internal perimeter at $\mathrm{x}=0: \mathrm{y}=0$

Line $\mathrm{AB}$, along the $\mathrm{x}$-axis, is assumed to be a circular arc in the $\mathrm{x}-\mathrm{z}$ plane. A given point, $\mathrm{F}$, subtends an angle $\varepsilon_{2}$ with the vertical. The value of $\varepsilon_{2}$ is constant around the X-circle, and $\varepsilon_{2}$ is a function of $\mathrm{X}$ only.

$\mathrm{X}=0$ is the centre of stress for the bulk, principal stress cap system. Symmetry is assumed, as in the Virtual Core of the previous model(Matchett et al, 2015). Therefore, at $X=0 ; \varepsilon_{2}=0$, or in terms of the previous model: $\beta_{\mathrm{c}}=0$

$\lambda$ is the slope of the $\mathrm{X}$-line as seen from the $\mathrm{y}$-axis. It is a projection of the path around the $\mathrm{X}$ line in 3-d space. The sense of the slope of the $\lambda$-line is the opposite of the original model (Matchett et al 2015). The slope is downwards with increasing $\mathrm{x}$ in this model, whereas it was upwards with $\mathrm{x}$ in Matchett et al(2015, 2016).

It is assumed that the $\psi_{1}$-line at the flow channel border describes a circular arc on the unrolled, flattened surface of the border. It has a length $\mathrm{P}_{\mathrm{f}}$ : the internal half perimeter of the flow channel:

$$
P_{f}=r_{1}\left(\pi / 2-\vartheta_{o}\right)
$$

The circular arc progresses across the flow channel perimeter from $\varepsilon_{2}=0$ to $\varepsilon_{2}=\beta_{\mathrm{w}}$, the wall normal angle. The arc has a radius $\mathrm{R}_{2 \theta}$, where:

$$
R_{2 \theta}=\frac{P_{f}}{\left(\sin \beta_{w}-\sin \beta_{c}\right)}=\frac{P_{f}}{\sin \beta_{w}}
$$

Thus, for a given value of $\mathrm{X}$ and therefore $\varepsilon_{2}$, the distance of the intersection below reference level $\mathrm{Z}, \mathrm{dZ}_{\theta}$ is:

$$
d Z_{\theta}=R_{2 \theta}\left(\cos \beta_{c}-\cos \varepsilon_{2}\right)=R_{2 \theta}\left(1-\cos \varepsilon_{2}\right)
$$

Likewise, the $\mathrm{x}$-direction displacement in negative $\mathrm{x}$-space, $\mathrm{dX} \mathrm{X}_{\theta}$ is given by:

$$
d X_{\theta}=r_{1}(1-\sin \theta)
$$

The geometry underpinning the calculation of $\lambda$ is shown in Figure 9. 


$$
\tan \lambda=\frac{d Z-d Z_{\theta}}{X+d X_{\theta}}
$$

The $\left(\varepsilon_{1}, \mathrm{X}, \mathrm{Z}\right)$ co-ordinate system can then be related to the $(\mathrm{x}, \mathrm{y}, \mathrm{z})$ system as follows:

$$
\begin{aligned}
& x=O+R_{1} \cos \varepsilon_{1} \\
& y=R_{1} \sin \varepsilon_{1} \\
& z=Z-\left(x+d X_{\theta}\right) \tan \lambda-d Z_{\theta}
\end{aligned}
$$

A typical principal stress cap surface is shown in Figure 10.

\section{Force Balances}

The changes in geometry are taken into account in the 3 force balance equations(Matchett et al 2015, 2016). The change in sense of $\lambda$ and hence $\varepsilon_{3}$ results in changes in sign of some of the terms in the equations, but fundamentally, the equations are as in the previous model:

$$
\begin{aligned}
& \frac{\partial}{\partial \varepsilon_{1}}\left[\sigma_{2} R_{2} \frac{\partial \varepsilon_{2}}{\partial X} \frac{\partial w_{2}}{\partial Z}\right]= \\
& -\sigma_{1} \frac{R_{1}}{\cos \varepsilon_{3}} \frac{\partial w_{2}}{\partial Z} E \frac{\partial \psi_{1}}{\partial X}+\sigma_{3}\left(\frac{R_{1}}{\cos \varepsilon_{3}}\right) R_{2} \frac{\partial \varepsilon_{2}}{\partial X} \frac{D \varepsilon_{3}}{D Z}-\rho g R_{1} R_{2} \frac{\partial \varepsilon_{2}}{\partial X} \frac{\partial w_{2}}{Z} \cos \varepsilon_{2} \tan \varepsilon_{3}
\end{aligned}
$$

$$
\begin{aligned}
& \frac{D}{D X}\left[\sigma_{1} \cos \varepsilon_{2}\right]= \\
& -\frac{\sigma_{1}}{R_{1} \cos \varepsilon_{3}}\left(\frac{\partial w_{1}}{\partial X}\right)\left(\frac{\partial w_{2}}{\partial Z}\right)+\sigma_{2}\left(\frac{R_{2}}{R_{1}}\right) E \frac{\partial \varepsilon_{2}}{\partial X} \frac{\partial w_{2}}{\partial Z}-\sigma_{3}\left(\frac{R_{2}}{\cos \varepsilon_{3}}\right) \frac{\partial \varepsilon_{2}}{\partial X} \frac{D \varepsilon_{2}}{D Z}+\rho g R_{2} \frac{\partial \varepsilon_{2}}{\partial X} \frac{\partial w_{2}}{\partial Z} \sin \varepsilon_{2}
\end{aligned}
$$

or

$$
\begin{gathered}
\frac{\partial}{\partial X}\left[\sigma_{1} \cos \varepsilon_{2}\right]=-\frac{\partial}{\partial \varepsilon_{1}}\left[\sigma_{1} \cos \varepsilon_{2}\right] \frac{\partial \psi_{1}}{\partial X} \\
-\frac{\sigma_{1}}{R_{1} \cos \varepsilon_{3}}\left(\frac{\partial w_{1}}{\partial X}\right)\left(\frac{\partial w_{2}}{\partial Z}\right)+\sigma_{2}\left(\frac{R_{2}}{R_{1}}\right) E \frac{\partial \varepsilon_{2}}{\partial X} \frac{\partial w_{2}}{\partial Z}-\sigma_{3}\left(\frac{R_{2}}{\cos \varepsilon_{3}}\right) \frac{\partial \varepsilon_{2}}{\partial X} \frac{D \varepsilon_{2}}{D Z}+\rho g R_{2} \frac{\partial \varepsilon_{2}}{\partial X} \frac{\partial w_{2}}{\partial Z} \sin \varepsilon_{2}
\end{gathered}
$$


$\frac{D}{D Z}\left[\frac{\sigma_{3}}{\cos \varepsilon_{3}}\right]=-\left[\frac{\sigma_{3}}{\cos \varepsilon_{3}}\right]\left(\frac{1}{R_{2}} \frac{\partial w_{2}}{\partial Z}+\frac{W}{R_{1}}\right)+\left(\frac{\sigma_{1}}{R_{2} \cos \varepsilon_{3}}\right) \frac{\partial w_{2}}{\partial Z}+\left(\frac{\sigma_{2}}{R_{1}}\right) \frac{\partial w_{2}}{\partial Z} \frac{\partial \varepsilon_{3}}{\partial \varepsilon_{1}}-\rho g \frac{\partial w_{2}}{\partial Z} \cos \varepsilon_{2}$

or

$$
\begin{aligned}
& \frac{\partial}{\partial Z}\left[\frac{\sigma_{3}}{\cos \varepsilon_{3}}\right]=-\frac{\partial}{\partial \varepsilon_{1}}\left[\frac{\sigma_{3}}{\cos \varepsilon_{3}}\right] \frac{\partial \psi_{1}}{\partial X} \frac{D X}{D Z}-\frac{\partial}{\partial X}\left[\frac{\sigma_{3}}{\cos \varepsilon_{3}}\right] \frac{D X}{D Z}-\left[\frac{\sigma_{3}}{\cos \varepsilon_{3}}\right]\left(\frac{1}{R_{2}} \frac{\partial w_{2}}{\partial Z}+\frac{W}{R_{1}}\right) \\
& +\left(\frac{\sigma_{1}}{R_{2} \cos \varepsilon_{3}}\right) \frac{\partial w_{2}}{\partial Z}-\left(\frac{\sigma_{2}}{R_{1}}\right) \frac{\partial w_{2}}{\partial Z} \frac{\partial \varepsilon_{3}}{\partial \varepsilon_{1}}-\rho g \frac{\partial w_{2}}{\partial Z} \cos \varepsilon_{2}
\end{aligned}
$$

A force balance is also conducted on the flow channel, using a Janssen approach(Janssen, 1895).

A vertical force balance over an incremental element $\mathrm{dZ}$ gives:

$$
\begin{aligned}
& \frac{d \sigma_{f v}}{d Z}=\left\{\frac{\tau_{f} P_{f}+\tau_{f w} P_{f w}}{A_{f}}\right\}-\rho g \\
& \tau_{f}=K_{f} \mu \sigma_{f h} \\
& \tau_{f w}=\mu_{w} \sigma_{f h w}
\end{aligned}
$$

$\mathrm{P}_{\mathrm{f}}$ is the internal flow channel half perimeter $\mathrm{m}$

$\mathrm{P}_{\mathrm{fw}}$ is the flow channel wall perimeter $\mathrm{m}$

$\mathrm{A}_{\mathrm{f}}$ is the flow channel half area $\mathrm{m}^{2}$

$\sigma_{\mathrm{fv}}$ is the flow channel vertical stress $\mathrm{Pa}$

$\sigma_{\text {fh }}$ is the flow channel horizontal, normal stress $\mathrm{Pa}$

$\tau_{\mathrm{f}}$ is the flow channel shear stress $\mathrm{Pa}$ (assumed to act upwards)

$\tau_{\mathrm{fw}}$ is the flow channel wall shear stress $\mathrm{Pa}$

$\mu$ is the internal coefficient of friction

$\mu_{\mathrm{w}}$ is the coefficient of wall friction

The incremental element is curved to follow the flow channel border. Areas are projected area onto the horizontal plane.

$$
\begin{aligned}
& A_{f}=\frac{1}{2} r_{2}^{2}\left(\theta_{o}-\sin \theta_{o}\right)+\frac{1}{2} r_{1}^{2}\left(\left(\frac{\pi}{2}-\theta_{o}\right)-\sin \left(\frac{\pi}{2}-\theta_{o}\right)\right) \\
& P_{f}=r_{1}\left(\frac{\pi}{2}-\theta_{o}\right) \\
& P_{f w}=r_{2} \theta_{o}
\end{aligned}
$$

A horizontal stress is given by:

$$
\sigma_{f h}=K_{f} \sigma_{f v}
$$


$\mathrm{K}_{\mathrm{f}}$ is the flow channel stress ratio: horizontal stress/vertical stress

\section{Boundary Conditions}

The changes in geometry require modifications in some of the boundary conditions.

Equation 19 requires a boundary condition in $Z$, referred to reference surface coordinate $Z_{0}$ :

$$
Z=Z_{o}: \sigma_{f v}=\sigma_{f v o}
$$

$\sigma_{\mathrm{fh}}$ interacts with the bulk to form a boundary condition in $\sigma_{2}$, equation 16 .

$$
\varepsilon_{1}=\pi-\theta: \sigma_{2}=\sigma_{f h}
$$

noting that for $\varepsilon_{1}>=\pi-\theta$ the conditions in the flow channel apply, and for $\varepsilon_{1}<\pi-\theta$, stresses are calculated using equations 16-19.

Other boundary conditions include(Matchett et al 2015, 2016):

Fort equation 18

$$
\begin{aligned}
& Z=Z_{o}: \sigma_{3}=\sigma_{3 o} \\
& X=X_{\max }: \frac{\partial \sigma_{3}}{\partial X}=\gamma_{1} \sigma_{3}
\end{aligned}
$$

And equation 17

$$
\begin{aligned}
& X=0: \frac{\partial \sigma_{1}}{\partial X}=0 \\
& X=0: \frac{\partial \sigma_{2}}{\partial X}=0 \\
& X=0: \sigma_{1}=\sigma_{2}
\end{aligned}
$$

\section{Integration of the force balance equations}

The force balance equations were integrated using a finite difference methodology, in a modified and improved qb64 program. Attention was paid to the stability of the numerical algorithms. Specifically, either forward or backward differences were used in integration of equation 18, dependent upon the sign and value of the coefficients of $\sigma_{3}$, to ensure all coefficients of $\sigma_{3}$ were positive.

This gave a fast and reliable program over a wide range of input parameters.

\section{Comparison of the two region model with the Eurocode 1 calculation}

A model simulation is compared to Eurocode 1 calculations in Figure 11. The model is able to reproduce the stress in the flow channel, but the bulk stresses are too small. For the silo conditions in Figure 11, this was the "best fit" that could be found. The bulk stresses could be 
increased by making $\beta_{\mathrm{c}}>0$, but this creates conceptual, mathematical and numerical problems and could not be accepted as a possible solution.

The internal stress structure is indicated in Figure 12, where the principal stresses are plotted along the x-axis. $\sigma_{3}$ is much greater than the other two principal stresses for most of the axis. This is reflected in the Conical Yield Function (CYF). In order to aid representation a Conical Yield Function Index is defined:

$$
\begin{aligned}
& \mathrm{CYFI}=1 \text { if } \mathrm{CYF}>=0 \\
& \mathrm{CYFI}=-1 \text { if } \mathrm{CYF}<0
\end{aligned}
$$

Figure 13 shows CYFI in $\mathrm{X}-\varepsilon_{1}$ space. Clearly, most of the bulk space is above the yield locus. Values of CYFI between +1 and -1 are an artifact of the surface plotting function in Excel, which was designed to model smooth surfaces.

Therefore, a system must be found in which the stresses transmitted to the bulk solids across the flow channel internal wall are increased. The flow channel cannot generate sufficient horizontal stress for the high, vertical stress within the central region of the bulk solids.

A transition zone is required. This is equivalent to the high stress zone in Eurocode 1(2006). It would be cynical to introduce an intermediate zone between the flow channel and the bulk solids just because there is one in Eurocode 1. However, initial modelling with a two region model has provided sound practical and conceptual reasons for a transition zone:

1. A transition zone provides a "buffer" between the relatively low stress regime of the flow channel and the high stresses within the central bulk region

2. The transition zone also provides a break between the dynamic flow channel and the static, steady state principal stress cap zone

3. The transition zone should allow sufficiently high values if $\sigma_{2}$ and $\sigma_{1}$ such that a greater proportion of the silo remains within the CYF at any depth

4. Horizontal force balances can be satisfied

\section{The three zone model for a wall flow channel}

The principal stress cap model can be modified to introduce a transition zone between the flow channel and the bulk solid principal stress cap zone.

This is conveniently done by introducing an inner circular arc, inside that marked out by angle $\theta_{\mathrm{o}}$. This has arc angle $\eta$ and half-chord length $\mathrm{l}_{\mathrm{f}}$ - Figure 14. Thus, the flow channel is now bounded by angle $\eta$, and the transition zone by angle $\theta_{0}$. 
A subscript convention has been employed whereby the first letter of the subscript denotes the zone:

subscript f-flow channel

subscript $\mathrm{t}$ - transition zone

The second letter refers to either:

$\mathrm{v}$ - vertical

h - horizontal

A subscript w denotes the silo wall, as opposed to an internal surface

Force balances on the flow channel are controlled by equations 19 and 20, but geometrical parameters are modified and the flow channel radius is now referred to as $r_{3}$ :

$$
\begin{aligned}
& A_{f}=\frac{1}{2} r_{2}^{2}(\eta-\sin \eta)+\frac{1}{2} r_{3}^{2}\left(\left(\frac{\pi}{2}-\eta\right)-\sin \left(\frac{\pi}{2}-\eta\right)\right) \\
& P_{f}=r_{3}\left(\frac{\pi}{2}-\eta\right) \\
& P_{f w}=r_{2} \eta \\
& r_{3}=r_{2} \tan \eta \\
& l_{f}=r_{2} \sin \eta=r_{3} \cos \eta
\end{aligned}
$$

The geometry of the transition zone is now described as:

$$
\begin{aligned}
& P_{t}=r_{1}\left(\frac{\pi}{2}-\theta_{o}\right) \\
& P_{t w}=r_{2}\left(\theta_{o}-\eta\right) \\
& A_{t}=\frac{1}{2} r_{2}^{2}\left(\theta_{o}-\sin \theta_{o}\right)+\frac{1}{2} r_{3}^{2}\left(\left(\frac{\pi}{2}-\theta_{o}\right)-\sin \left(\frac{\pi}{2}-\theta_{o}\right)\right)-A_{f}
\end{aligned}
$$

Where:

$\mathrm{Pt}$ is the internal half-perimeter between the transition zone and the bulk $\mathrm{m}$ $\mathrm{P}_{\mathrm{tw}}$ is the wall perimeter of the transition zone $\mathrm{m}$ $A_{t}$ is the cross section area of the transition zone $\mathrm{m}^{2}$

Figure 15 shows the stresses acting upon the transition zone element $\mathrm{dZ}$. It is assumed that the interface between the transition zone and the bulk is a plane of principal stress, such that the horizontal principal stress is transmitted across the interface into the bulk: $\sigma_{2 b}$ in Figure 15. This acts as a boundary condition - as in equation 22. There are no shear forces on this interface. 
The assumption of principal stresses acting on the border of the transition zone is made for a number of reasons:

- It is necesssary for implementation of the principal stress cap model

- The borders between the zones are arbitrary - Eurocode 1 suggests calculations for at least 3 flow channel sizes - Eurocode 1(2016), page 53

- Therefore, it is reasonable to select a location of boundary which is a principal stress plane, or a close approximation

The vertical force balance is given by:

$$
\frac{\partial \sigma_{t v}}{\partial Z}=\frac{\left(-\tau_{f} P_{f}+\tau_{t w} P_{t w}\right)}{A_{t}}-\rho g
$$

The horizontal force balance is:

$$
\sigma_{t h w}=\frac{\left(\sigma_{t h} l_{o}-\sigma_{f h} l_{f}\right)}{\left(l_{o}-l_{f}\right)}
$$

$\sigma_{\mathrm{tv}}$ is the vertical stress in the transition zone $\mathrm{Pa}$

$\sigma_{\mathrm{th}}$ is the horizontal stress in the transition zone $\mathrm{Pa}$

$\sigma_{\text {thw }}$ is the horizontal stress at the wall in the transition zone $\mathrm{Pa}$

$\tau_{\mathrm{tw}}$ is the wall shear stress in the transition zone $\mathrm{Pa}$

and

$$
\begin{aligned}
& \sigma_{t h}=K_{t} \sigma_{t v} \\
& \tau_{t w}=\mu_{t w} \sigma_{t h} \\
& \sigma_{2 b}=K_{t b} \sigma_{t h}
\end{aligned}
$$

$\mathrm{K}_{\mathrm{t}}$ is the averaged stress ratio constant for the vertical force balance

$\mathrm{K}_{\mathrm{tb}}$ is the averaged force ratio for the horizontal the force balance

The $\mathrm{K}$ and $\mu$ values are not necessarily limiting values.

A negative value of $\mu$ indicates that the force acts in the opposite direction to that shown in Figure 15. It is primarily a directional device. The direction of $\tau_{\mathrm{f}}$ shown in Figure 15 is equivalent to an upwards, vertical shear stress acting upon the material in the flow channel. A reversal of direction implies rotation within the flow channel, which is completely compatible with the assumption of frictional flow.

The transition/bulk boundary is curved to follow the line of principal stress as in equation 11. The wall and flow channel boundaries are horizontally planar. 


\section{Wall normal stress behaviour of the three-zone model}

Typical wall normal stress behaviours are shown in Figure 16. The flow channel and transition zone are clearly present.

The wall normal stress in the bulk zone is not constant(unlike Eurocode 1). The stress drops in the vicinity of the transition zone boundary. It then rises with $\beta_{\mathrm{w}}>0$, equivalent to passive stress with a convex principal stress cap in the bulk, or passes through a local maximum and then falls for $\beta_{\mathrm{w}}<0$, equivalent to an active stress with a concave principal stress cap in the bulk, as $\varepsilon_{1}$ decreases - see Matchett et al(2016).

\section{Comparison of the three-zone model with stress predictions for Chen et al(2007) experimental data}

Chen et al(2007) conducted extensive tests on a full-size circular silo. Figure 16 of their paper shows extensive wall normal stress data at incipient flow. This has been calculated from a large array of strain gauges mounted on the silo wall. The conditions and materials used are shown in Table 1.

The data of Chen et al(2007) is presented as percent change from the steady stress values. In order to convert to actual stresses, the data were scaled using the stresses calculated from the Eurocode 1 calculation procedure. The range of peak data were scaled by the range between flow channel stress and high stress/transition zone stress of Eurocode 1.

The Chen et al(2007) data show complex patterns. In general, there is a low, minimum stress at the centre of the flow channel $\left(\varepsilon_{1}=180^{\circ}\right)$. The stress then increases rapidly with azimuthal angle(decrease in $\varepsilon_{1}$ ) to a peak corresponding to the high stress/transition zone. It then changes through a series of oscillation. Rings A, C and D have a tendency to a general decrease in stress around the circumference, whereas B shows a tendency to increase. It is suggested that the reader study Figure 16 in the original paper(Chen et al, 2007) to appreciate the details and complexities of the data. The lines presented in this paper are quantitative trend lines taken from the original.

The data have been fitted to the model "by eye", and three simulations were performed. The three simulations are shown in Figures 17. Values of parameters are given in Table 2.

A statistical approach has not been used for several reasons:

- a great deal of effort would be required for a questionable or insignificant gain 
- there are a number of criteria that could be used to generate a fit: overall data comparison: an optimisation of some or just one of the three zones e.g best fit the bulk zone only

- these data were fitted to the model by prioritising the prediction of flow channel and transition stresses in S1 and S2. The bulk stress region was the adjusted without changing the other values, by adjusting wall angle $\beta_{\mathrm{w}}$ and wall gradient factor $\gamma_{1}$

- The limiting value of wall friction was used for the channel: $\mu_{\mathrm{fw}}$ 
Table 1 The conditions of Chen et al(2007) experimental data

\begin{tabular}{|c|c|}
\hline Silo properties & \\
\hline Silo diameter & $4.2 \mathrm{~m}$ \\
\hline Barrel section height & $9.5 \mathrm{~m}$ \\
\hline Measurement ring depth: & \\
\hline A & $3.5 \mathrm{~m}$ \\
\hline B & $5 \mathrm{~m}$ \\
\hline $\mathrm{C}$ & $6.5 \mathrm{~m}$ \\
\hline $\mathrm{D}$ & $8 m$ \\
\hline \multicolumn{2}{|l|}{ Bulk Material Properties } \\
\hline \multicolumn{2}{|l|}{ Material used: iron ore pellets } \\
\hline bulk density & $2300 \mathrm{~kg} / \mathrm{m}^{3}$ \\
\hline angle of wall friction & $26.2^{\circ}$ \\
\hline angle of internal friction & $52^{\circ}$ \\
\hline Limiting coefficient of wall friction & 0.5 \\
\hline Limiting coefficient of internal friction & 0.91 \\
\hline Limiting principal stress ratio(Mohr Circle) & 8.2 \\
\hline Further details in Rotter et al(1995) & \\
\hline
\end{tabular}

Table 2 Values of parameters used in simulations of the Chen et al(2007) data

\begin{tabular}{|c|c|c|c|c|c|c|c|c|c|c|c|}
\hline $\begin{array}{c}\text { Simulation } \\
\text { Number }\end{array}$ & $\begin{array}{c}\boldsymbol{\theta}_{\mathbf{o}} \\
\mathbf{d e g}\end{array}$ & $\begin{array}{c}\eta \\
\mathbf{d e g}\end{array}$ & $\boldsymbol{\mu}$ & $\boldsymbol{\mu}_{\mathbf{f w}}$ & $\boldsymbol{\mu}_{\mathrm{tw}}$ & $\mathbf{K}_{\mathbf{f}}$ & $\mathbf{K}_{\mathbf{t}}$ & $\mathbf{K}_{\mathbf{t b}}$ & $\boldsymbol{\beta}_{\mathbf{w}}$ & $\gamma_{\mathbf{1}}$ & \\
$\mathbf{d e g}^{-1}$ & $\mathbf{m}^{-1}$ & & & & & & & & \\
\hline $\mathbf{S 1}$ & 32.7 & 20.8 & -0.11 & 0.5 & 0.25 & 3 & 0.325 & 0.175 & -4 & 0.1 & \\
\hline $\mathbf{S 2}$ & 32.7 & 20.8 & -0.11 & 0.5 & 0.25 & 3 & 0.325 & 0.175 & 15 & -30 & \\
\hline $\mathbf{S 3}$ & 32.7 & 20.8 & -0.11 & 0.5 & 0.25 & 3 & 0.365 & 0.175 & 17 & -30 & \\
\hline
\end{tabular}

Simulation Number 1(S1): the model outputs were fitted to data for all rings A, B, C and D. The parameters of the flow channel and transition zone were adjusted to give a good prediction of the experimental data. The principal stress cap parameters $\beta_{\mathrm{w}}$ and $\gamma_{1}$ were then 
adjusted to give a fit to the experimental data. This resulted in a value of $\beta_{\mathrm{w}}$ of $-4^{\mathrm{o}}$ giving a concave principal stress cap with an active state of stress.

The value of $\beta_{\mathrm{w}}$ was selected because it is the smallest magnitude negative value that produces bulk zone stresses within the Conical Yield Locus(CYF). Below $-4^{\mathrm{o}}$, areas of the bulk zone which exceed the CYF begin to appear.

This is the best overall simulation of those presented, giving reasonable agreement, both qualitatively and quantitatively over the complete experimental data set of four rings.

Simulation Number 2(S2): the data were fitted to experimental data from Rings A and B only, giving priority to prediction of flow channel and transition zone stresses. A passive stress system was chosen in the bulk zone with $\beta_{\mathrm{w}}>0$ : $\beta_{\mathrm{w}}=15^{\circ} \cdot \beta_{\mathrm{w}}=15^{\circ}$ was chosen as the maximum value with all of the bulk zone remaining within the CYF.

Simulation Number 3(S3): conditions were modified from S2 to fit the whole of the three zones to the experimental data for Rings A and B only. Accuracy of prediction in the flow channel and transition zones was sacrificed for closer correspondence to experimental data in the bulk zone.

\section{Discussion}

There have been several problems in the development of this model. In conceptual terms, the principal stress cap model is explicitly static, but is being used to model an essentially dynamic system of channel flow. The use of a transition zone, as a barrier between the dynamic flow channel zone and the static bulk has had some success in overcoming the problems. The purposes of the transition zone are:

1. to shelter the relatively low stress flow channel from the high stress region around the centre of the silo - see Figure 11

2. to allow high stresses at the transition/bulk interface, independent of the low stresses in the flow zone, thereby keeping stresses within the yield locus

3. to form a transition between the dynamic flow channel and the static bulk

4. to allow a transition from the passive stress state in the flow channel to the active stress state in the bulk

The transition zone contains complex stress systems, ranging from active at one boundary, to passive at the other. It can be argued that it is the most challenging of the three zones to analyse. It might even be said that all the really difficult problems have been lumped into the 
transition zone and ignored, but they are really beyond the scope of this paper and a topic for future work. This is a challenging area for further research.

Lack of experimental data hinders the modelling effort.

There are many issues to be considered when fitting silo models to experimental data, as discussed in Matchett et al(2016). There are major problems in trying to infer internal principal stress orientations from wall stress readings and vice versa. The authors propose that these problems are generic and not confined to the present model. For example, in the Janssen(1895) equation, the Janssen coefficient is given by:

$$
b_{\text {Janssen }}=\frac{4 \mu_{w} K}{d}
$$

$\mathrm{K}$ is the ratio of horizontal to vertical stress

$\mu_{\mathrm{w}}$ is the coefficient of wall friction

$\mathrm{d}$ is the silo diameter $\mathrm{m}$

The Janssen coefficient can be determined from experimental wall stress data, from which the product $\mathrm{K} \mu_{\mathrm{w}}$ can be found. Neither $\mathrm{K}$ nor $\mu_{\mathrm{w}}$ can be found individually without additional information: from the data the internal and wall properties inherent in Janssen cannot be found without further information. For example, the coefficient of wall friction could be measured externally and assumed to be at the limit. Similarly, vertical wall friction could be measured within the silo, as well as normal stress, giving an "in-situ" value of $\mu_{\mathrm{w}}$. However, if one were to question the validity of the Janssen assumption that vertical stress is constant across the horizontal section of the silo(ie internal stress structure), neither of these measurements would provide insights.

In the context of the approach used in this paper, the proposed model is not the only principal stress cap geometry that could be proposed. The present model uses boundary conditions as a basis for interpolation within the cross section of the silo. It is a natural development of previous models(Matchett et al 2015, 2016) and is amenable to mathematical analysis. But it is not unique. As far as the authors are aware, there are no experimental data of internal stress distributions with which to calibrate this, or any other internal, structural model.

$\mathrm{S} 1$ is a reasonable qualitative fit to the data over its full range. The stresses in the flow channel and transition zone are accurately predicted - within +/- $200 \mathrm{~Pa}$. Overall, the 
difference between the model and Chen et al data has a mean value of $581 \mathrm{~Pa}$ with 0.05 confidence limits of $1310 \mathrm{~Pa}$. This is reasonable for data with a maximum value of $24000 \mathrm{~Pa}$. There are discrepancies at the borders between the flow channel/transition zone and transition zone/bulk due to the simplifications of the Janssenian models used, and the location of the borders can have a great effect upon the apparent goodness of fit of the data. For example, if the data points close to the transition zone border are eliminated(these are the points where the difference is greatest) the mean difference between model and data is $-195 \mathrm{~Pa}$ with a 0.05 confidence level of $666 \mathrm{~Pa}$. It is clear that the conditions within the flow and transition zones are simplifications, and that location of borders between zones is arbitrary.

Wall normal stress at the transition/bulk border is relatively low, followed by a general rapid increase in stress in the border region as $\varepsilon_{1}$ decreases, from a relatively low to a maximum bulk zone value. The stress then decreases as $\varepsilon_{1}$ tends to zero. This is in qualitative agreement with the experimental data, except perhaps for Ring B.

The model does not predict the oscillations present in the experimental. Likewise, it could not predict dynamic phenomena, such as shown in Figure 15 of the Chen et al(2007) paper. The oscillations within the bulk zone could be due to a number of causes:

- transmission of stress waves originating within the shear zone, due to flow phenomena and slip.

- discontinuities due to slip and rupture zones within the bulk zone

Unfortunately, the exact causes cannot be determined without further information. This is clearly an aspect of Eurocode 1 that requires further experimental investigation.

S1 has not been optimised, other than "by eye". It could form the starting point of a systematic minimisation of differences. The parameters of S1 apply reasonably well over the full extent of Rings A, B, C and D both qualitatively and quantitatively. The model can scale both azimuthally and with depth, with an active stress state in the bulk zone.

Therefore, the principal stress cap surface model can be applied to systems with a flow channel adjacent to the silo wall and model the bulk solids zone reasonably well.

However, many issues remain:

- Why are data from Ring B somewhat different to the others, with rising/steady bulk zone stresses as $\varepsilon_{1}$ tends to zero.

- The greatest deviations are in the region of the transition/bulk zone interface. Part of this is due to the selection of Janssen(1895)-type models for the flow and transition zones and the choice of the location of the borders. The exclusion of the large 
difference data points from the statistics greatly reduces the magnitude of difference between the model and the data. Models with greater detail are required for these zones and the nature and location of the borders requires further investigation, if indeed, the zones actually exist other than as a theoretical convenience.

- The oscillations inherent in the data are not present in the model. Are they dynamic in nature?

- To what extent does experimental error influence these data?

Clearly, it would be extremely useful to have many more data sets of the extensive nature of Chen et al(2007), to help answer these points.

The stress systems in the present model are very complex, and agreement between model and data in one part of the silo does not imply universal applicability. S2 and S3 have been presented as a case study of potential pitfalls. S2 shows agreement in stresses within the flow channel and transition zone, as S1, but with a passive(convex principal stress cap)) bulk zone. The bulk zone stress for S2 is approximately an upper envelope for the experimental data for Rings A and B only.

S3 is a development of S2 in which accuracy of prediction of flow channel and transition zone wall normal stresses has been sacrificed somewhat in order to provide a better fit between model and data in the bulk zone, again for Rings A and B only.

In the absence of other information, either of these simulations might be considered acceptable.

However, when the simulations are plotted against the experimental data for Rings C and D, it can be seen that the models show poor qualitative and quantitative agreement. In particular, the model shows an increase in wall stress as $\varepsilon_{1}$ decreases towards zero, while the experimental data decrease as $\varepsilon_{1}$ tends to zero. Therefore, S2 and S3 can be used as empirical predictors in the region of Rings A and B, but are not more widely applicable.

A modeller might find reasons for rejecting the data from Rings $C$ and $D$ :

- $\mathrm{C}$ and $\mathrm{D}$ are nearer the surface and might be suffering from surface effects

- The stresses might not be fully developed in the region of C and D

These arguments would be plausible if it were not for the fact that an active bulk zone(S1) describes the full extent of these data.

Therefore, it may be deduced that large data sets are required to calibrate and test models thoroughly. Comparison with limited data sets will give limited validity and produce only 
empirical relationships valid over a restricted range of conditions. Limited data may suggest misleading models of internal stress structure. Data points which do not agree with a proposed model should not be arbitrarily rejected unless there is evidence to justify such rejection. A corollary of this argument is that extrapolation beyond data limits can be very misleading.

However, even for S1, it is wise to remember to old adage "just because a model can be fitted to experimental that does not necessarily mean that it is "true". We always need more evidence.

Finally, this study of wall flow channels has made the authors appreciate the quality of the analysis inherent in Eurocode 1. The authors of Eurocode 1 have taken a tremendously complex stress situation and developed a design procedure that encapsulates and simplifies into a workable set of procedures.

\section{Conclusions}

A flow channel in the vicinity of the wall of a cylindrical silo, as described in Eurocode 1(2006), has been modelled by a three-zone model, consisting of:

- the flow channel

- the transition zone

- the bulk of the solids

The flow channel and the transition zone were modelled by Janssen-type equations. The bulk solid was modelled by the principal stress cap surface approach.

The paper has shown that the principal stress cap surface approach can be applied to this type of system.

The model was compared and calibrated against experimental data from a full scale silo by Chen et al(2007). The model was able to describe the data both axially and azimuthally reasonably well.

An illustration of the pitfalls of comparing wall stress data with models has been shown in reference to the model and the Chen data.

Large experimental data sets are required to calibrate and test a model. Furthermore, unfortunate data points cannot be arbitrarily rejected.

Improved models of the flow channel and transition zone are needed. 
More experimental data are required to calibrate existing models and help develop new models.

\section{References}

Chen J.F., J.M.Rotter, J.Y.Ooi, Z.Zhong, 2007, Correlation between the flow pattern and wall pressures in a full scale experimental silo. Engineering Structures, 29, 2308-2320

Enstad G., 1975, On the theory of arching in mass flow hoppers, Chem.Eng.Sci. 30, 12731283

Eurocode 1, EN 1991-4, 2006. Action on structures - Part 4: Silos and Tanks. Brussels, Belgium.

Janssen H.A., 1895. Versuche uber getreidedruck in silozellen, Zeitschrift verein Deutsche Inginieur, 39,1045-1049

Lapko A., 2010, Pressure of agricultural bulk solids under eccentric discharging of cylindrical concrete silo bin. International Agrophysics, 24, 51-56

Matchett A.J., P.A.Langston, D.McGlinchey. 2015. A model for stresses in a circular silo with an off-centre circular core, using the concept of a principal stress cap: Solutions for a completely filled silo and comparison with DEM data. Chemical Engineering Research and Design 93, 330-348

Matchett A.J., P.A.Langston, D.McGlinchey. 2016. A "principal stress cap" model for stresses in a circular silo with an off-centre circular core: Finite core models, including filled silos, incipient flow and switch stresses. Chemical Engineering Research and Design 106, 263-282

Nedderman R.M. 1992, Statics and Kinematics of Granular Materials, Cambridge University Press, Cambridge, England

Rotter J.M, J.Y.Ooi, I.F.Chen, P.J.Tiley, I.McIntosh, F.R.Bennett. 1995. Flow pattern measurement in full scale silos. British Materials Handling Board Publications, London.

Sielamovicz I., M.Czech, T.A Kowalewski. 2010. Empirical description of flow parameters in eccentric flow inside a silo model, Powder Technology 198, 381-394

Sielamovicz I., M.Czech, T.A Kowalewski. 2011. Empirical analysis of eccentric flow registered by the DPIV technique inside a model silo, Powder Technology 212, 38-56

Sielamovicz I., M.Czech, T.A Kowalewski. 2015. Comparative analysis of empirical descriptions of eccentric flow in silo model by the linear and non-linear regressions, Powder Technology 270, 393-410 


\section{Notation}

The notation used in Matchett et $\mathrm{al}(2015,2016)$ is used in this paper. Additional notation used specifically in this paper is given below:

\begin{tabular}{|c|c|c|}
\hline SYMBOL & DESCRIPTION & UNITS \\
\hline $\mathrm{A}_{\mathrm{f}}$ & flow channel zone cross section area & $\mathrm{m}^{2}$ \\
\hline $\mathrm{A}_{\mathrm{t}}$ & transition zone cross section area & $\mathrm{m}^{2}$ \\
\hline $\mathrm{b}_{\text {Janssen }}$ & Janssen coefficient & $\mathrm{m}^{-1}$ \\
\hline CYFI & Conical Yield Function Index & - \\
\hline $\mathrm{d}$ & silo diameter & $\mathrm{m}$ \\
\hline$\overline{\mathrm{K}_{\mathrm{f}}}$ & flow channel zone stress ratio: horizontal/vertical & - \\
\hline $\mathrm{K}_{\mathrm{t}}$ & transition zone stress ratio: horizontal/vertical & - \\
\hline $\mathrm{K}_{\mathrm{tb}}$ & $\begin{array}{l}\text { transition zone stress ratio: horizontal/vertical, used in horizontal } \\
\text { force balance }\end{array}$ & - \\
\hline 1 & half chord length subtended by angle $\theta$ & $\mathrm{m}$ \\
\hline $1_{\mathrm{o}}$ & half chord length subtended by angle $\theta_{\mathrm{o}}$ : transition zone & $\mathrm{m}$ \\
\hline $\mathrm{l}_{\mathrm{f}}$ & flow channel half chord length & $\mathrm{m}$ \\
\hline$\overline{P_{f}}$ & flow channel half perimeter & $\mathrm{m}$ \\
\hline $\mathrm{P}_{\mathrm{fw}}$ & flow channel wall perimeter & \\
\hline$r_{3}$ & flow channel radius & $\mathrm{m}$ \\
\hline$\theta$ & $\begin{array}{l}\text { angle subtended between the silo centre and a point at the bulk } \\
\text { solid zone/transition zone boundary }\end{array}$ & - \\
\hline$\theta_{\mathrm{o}}$ & $\begin{array}{l}\text { angle subtended between the silo centre and the point where the } \\
\text { bulk solid zone boundary meets the silo wall }\end{array}$ & - \\
\hline$\mu$ & internal coefficient of friction & - \\
\hline$\mu_{\mathrm{w}}$ & coefficient of wall friction & - \\
\hline$\mu_{\mathrm{tw}}$ & transition zone coefficient of wall friction & - \\
\hline$\sigma_{\mathrm{fh}}$ & flow channel horizontal stress & $\mathrm{Pa}$ \\
\hline$\sigma_{\mathrm{fv}}$ & flow channel vertical stress & $\mathrm{Pa}$ \\
\hline
\end{tabular}




\begin{tabular}{|c|l|c|}
\hline$\sigma_{\mathrm{th}}$ & transition zone horizontal stress & $\mathrm{Pa}$ \\
\hline$\sigma_{\mathrm{thw}}$ & transition zone horizontal wall stress & $\mathrm{Pa}$ \\
\hline$\sigma_{\mathrm{tv}}$ & transition zone vertical stress & $\mathrm{Pa}$ \\
\hline$\sigma_{2 \mathrm{~b}}$ & $\begin{array}{l}\text { principal stress } \sigma_{2} \text { transmitted across the transition/bulk interface: } \\
\text { boundary condition for the bulk zone }\end{array}$ & $\mathrm{Pa}$ \\
\hline$\tau_{\mathrm{f}}$ & flow channel internal shear stress & $\mathrm{Pa}$ \\
\hline$\tau_{\mathrm{fw}}$ & flow channel wall shear stress & $\mathrm{Pa}$ \\
\hline & & \\
\hline
\end{tabular}

The notation used in the previous papers (Matchett et al 2015, 2016) is given below:

\begin{tabular}{|c|c|c|}
\hline SYMBOL & DESCRIPTION & UNITS \\
\hline $\mathrm{a}_{1}$ & Relates projected circle radius $\mathrm{R}_{1}$ to $\mathrm{X} \quad R_{1}=a_{1} X+a_{1}{ }^{\prime}$ & - \\
\hline$a_{1}{ }^{\prime}$ & Constant relating $\mathrm{R}_{1}$ to $\mathrm{X}-$ see $\mathrm{a}_{1}$ & $\mathrm{~m}$ \\
\hline $\mathrm{a}_{2}$ & Differential of projected circle centre $a_{2}=\partial O_{X} / \partial X$ & - \\
\hline $\mathrm{D}$ & $\begin{array}{l}\mathrm{D} / \mathrm{DX} \text { and } \mathrm{D} / \mathrm{DZ} \text { are differentials along the principal stress paths } \\
\text { for changes in } \mathrm{X} \& \mathrm{Z} \text { respectively }\end{array}$ & \\
\hline $\mathrm{e}_{1}$ & Angle used in the calculation of $\mathrm{R}_{2}$ & $\mathrm{rad}$ \\
\hline $\mathrm{E}$ & $\begin{array}{l}\text { Factor relating rotation in the horizontal plane to rotation on the } \lambda \text { - } \\
\text { plane }\end{array}$ & - \\
\hline $\mathrm{h}$ & Inner circle offset & $\mathrm{m}$ \\
\hline & & - \\
\hline $\mathrm{k}$ & Ratio of wall vertical to normal stress: Janssen model & - \\
\hline $\mathrm{K}_{\mathrm{w}}$ & Ratio of $\sigma_{1} / \sigma_{3}$ at the wall & - \\
\hline $\mathrm{M}$ & Conical Yield Function parameter & - \\
\hline $\mathrm{r}_{1}$ & Inner circle radius & $\mathrm{m}$ \\
\hline $\mathrm{r}_{2}$ & Outer circle (silo) radius & $\mathrm{m}$ \\
\hline $\mathrm{R}_{1}$ & Radius of projected horizontal circle of principal stress path & $\mathrm{m}$ \\
\hline $\mathrm{R}_{2}$ & Radius of principal stress cap at a general point & $\mathrm{m}$ \\
\hline $\mathrm{R}_{20}$ & Value of $R_{2}$ at $\varepsilon_{1}=0$ & $\mathrm{~m}$ \\
\hline $\mathrm{R}_{2 \pi}$ & Value of $\mathrm{R}_{2}$ at $\varepsilon_{1}=\pi$ & $\mathrm{m}$ \\
\hline
\end{tabular}




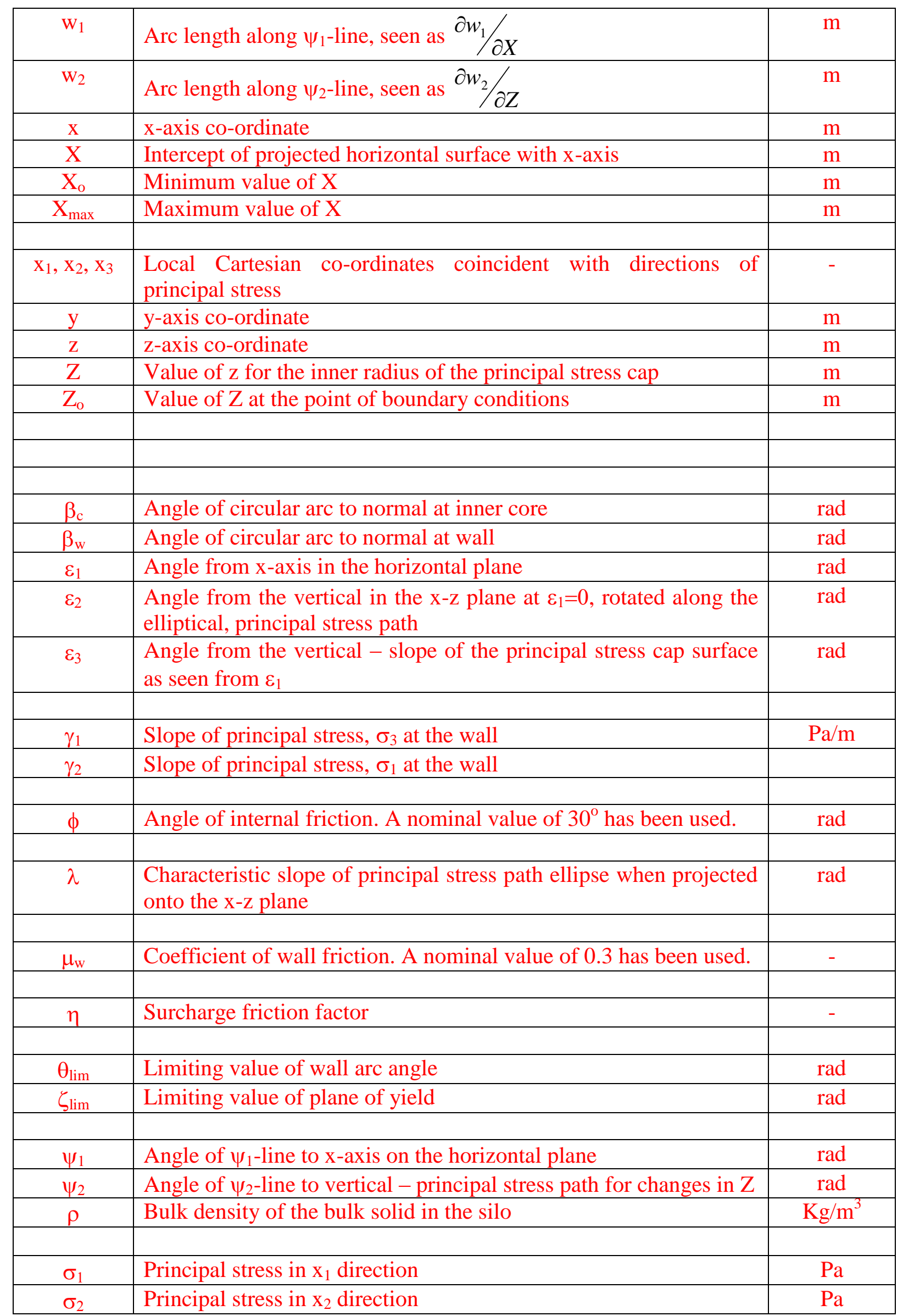




\begin{tabular}{|c|l|c|}
\hline$\sigma_{3}$ & Principal stress in $\mathrm{x}_{3}$ direction & $\mathrm{Pa}$ \\
\hline & & \\
\hline & & \\
\hline & & \\
\hline & & \\
\hline
\end{tabular}

Note that Figure 1 andFigure 2 are also additions to the paper 


\section{Figures}

Figure 1 The principal stress cap and essential structure of the principal stress cap, eccentric silo mod

Figure 2 Constant $X$-lines and corresponding $\psi_{1}$ - lines, projected onto the horizontal plane $\mathrm{X}$-lines represent the paths of azimuthal principal stress $\sigma_{2}$. The $\psi_{1}$ lines are the paths of radial stress $\sigma_{1}$

arbitrary scale; radii are full lines; $\psi_{1}$ lines are heavy, dashed lines; the dotted line is a line of constant $\varepsilon_{1}$

Figure 3 Wall normal stress data calculated by Eurocode 1 for a flow channel next to the silo wall

Figure 4 The conventional picture of a wall flow channel

Figure 5 Cross-section of the region of fully developed stress, showing the flow channel, which is circular in section and crosses the silo wall at right-angles

Figure 6 General azimuthal projected principal stress circle $X$

Figure $7 \quad \mathrm{X}$ and $\psi_{1}$ lines projected onto a horizontal plane.

$r_{2}=2 ; \theta_{0}=20^{\circ} ; 1_{0}=0.684 m ; r_{1}=0.728 m$

Figure 8 Plan view(top) and side view from the direction of the y-axis of the principal stress cap surface.

Figure 9 View of the stress cap surface and $\lambda$-line at $X$, seen from the $y$-axis. The calculation of $\lambda$, showing offsets in $\mathrm{X}$ and $\mathrm{Z}$ associated with the flow channel geometry.

Figure 103 -d plot of a principal stress cap half-surface for the Eurocode 1 model. $\mathrm{r}_{2}=4 \mathrm{~m} ; \beta_{\mathrm{w}}=30^{\circ} ; \beta_{\mathrm{c}}=0$

Figure 11 Comparison of wall normal stress for the two region model and Eurocode 1 calculations.

$\mathrm{r}_{2}=4 \mathrm{~m} ; \beta_{\mathrm{w}}=10^{\circ} ; 1_{\mathrm{o}}=1.2 \mathrm{~m} ; \mathrm{r}_{1}=1.26 \mathrm{~m} ; \mu=0 ; \mu_{\mathrm{w}}=0.2 ; \mathrm{Kw}=0.3$

Figure 12 Variation in principal stresses along the $\mathrm{x}$-axis; $\varepsilon_{1}=0$

Condition as in the simulation in Figure 9

Figure 13 Conical Yield Function Index in $\mathrm{X}-\varepsilon_{1}$ space for conditions in Figure 9

Figure 14 Three zone model for a flow channel in the vicinity of the silo wall, showing the transition zone

Figure 15 Force balance on the transition zone

Figure 16 General wall normal stress response for the three-zone model $\mathrm{r}_{2}=4 \mathrm{~m} ; 1_{\mathrm{o}}=1.6 \mathrm{~m} ; \mathrm{l}_{\mathrm{f}}=1.42 \mathrm{~m} ; \mu=-0.1 ; \mu_{\mathrm{w}}=0.25 ; \mu_{\mathrm{tw}}=0.125 ; \mathrm{K}_{\mathrm{f}}=3 ; \mathrm{K}_{\mathrm{t}}=2 ; \mathrm{K}_{\mathrm{tb}}=1$ $\beta_{\mathrm{w}}=10$ and $\gamma_{1}=-1$ or $\beta_{\mathrm{w}}=-10$ and $\gamma_{1}=1$

Figure 17 Comparison of Chen et al data(2007) with model predictions. A "best-fit" of data to the model.

Figure 17a Ring A, depth 8m

Figure 17b Ring B, depth $6.5 \mathrm{~m}$ 
Figure 17c Ring C, depth 5m

Figure $17 \mathrm{~d} \quad$ Ring D, depth $3.5 \mathrm{~m}$

See Table 1 for silo and material properties

See Table 2 for model parameters 


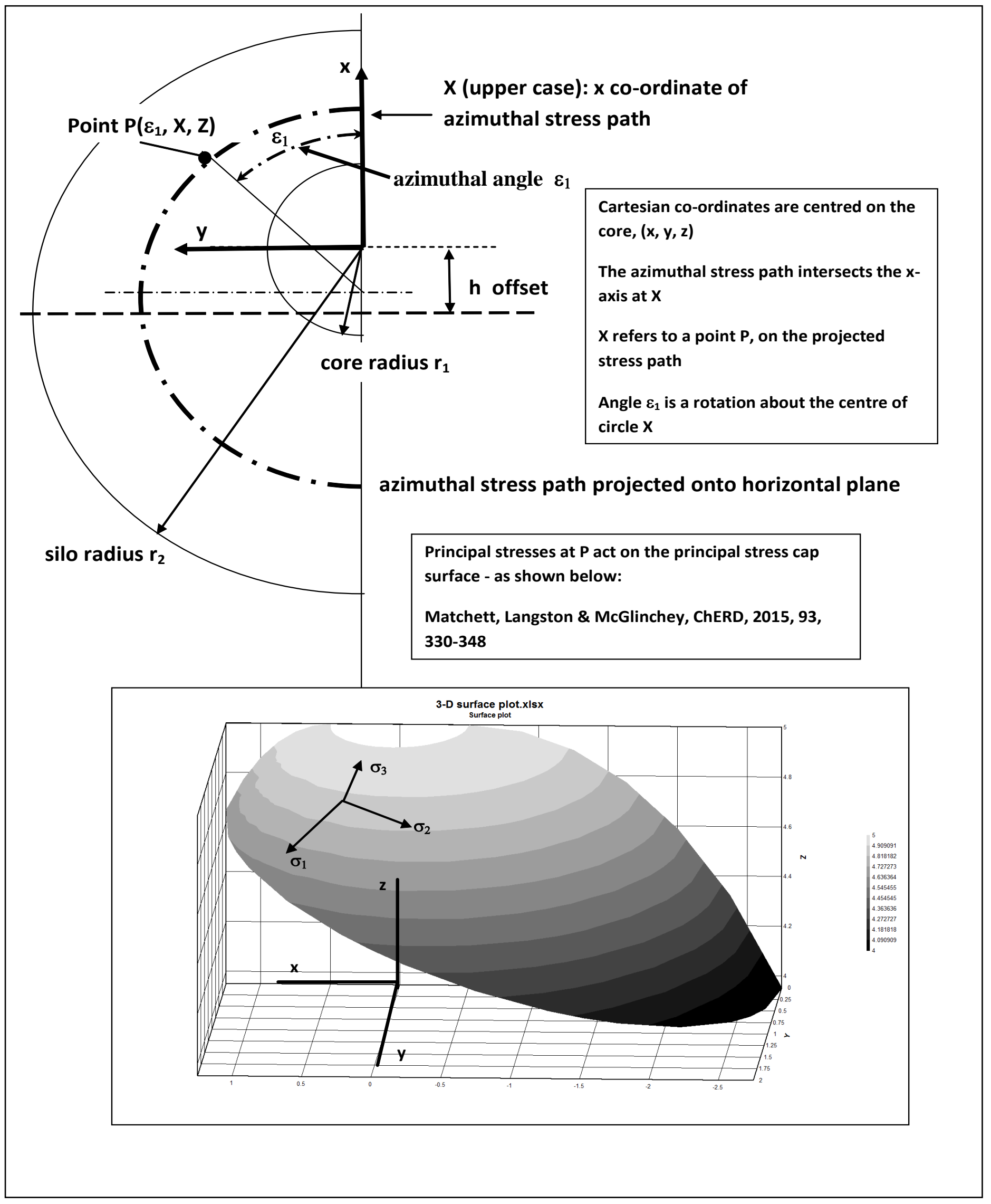

Figure 1 The principal stress cap and essential structure of the principal stress cap, eccentric silo mode 


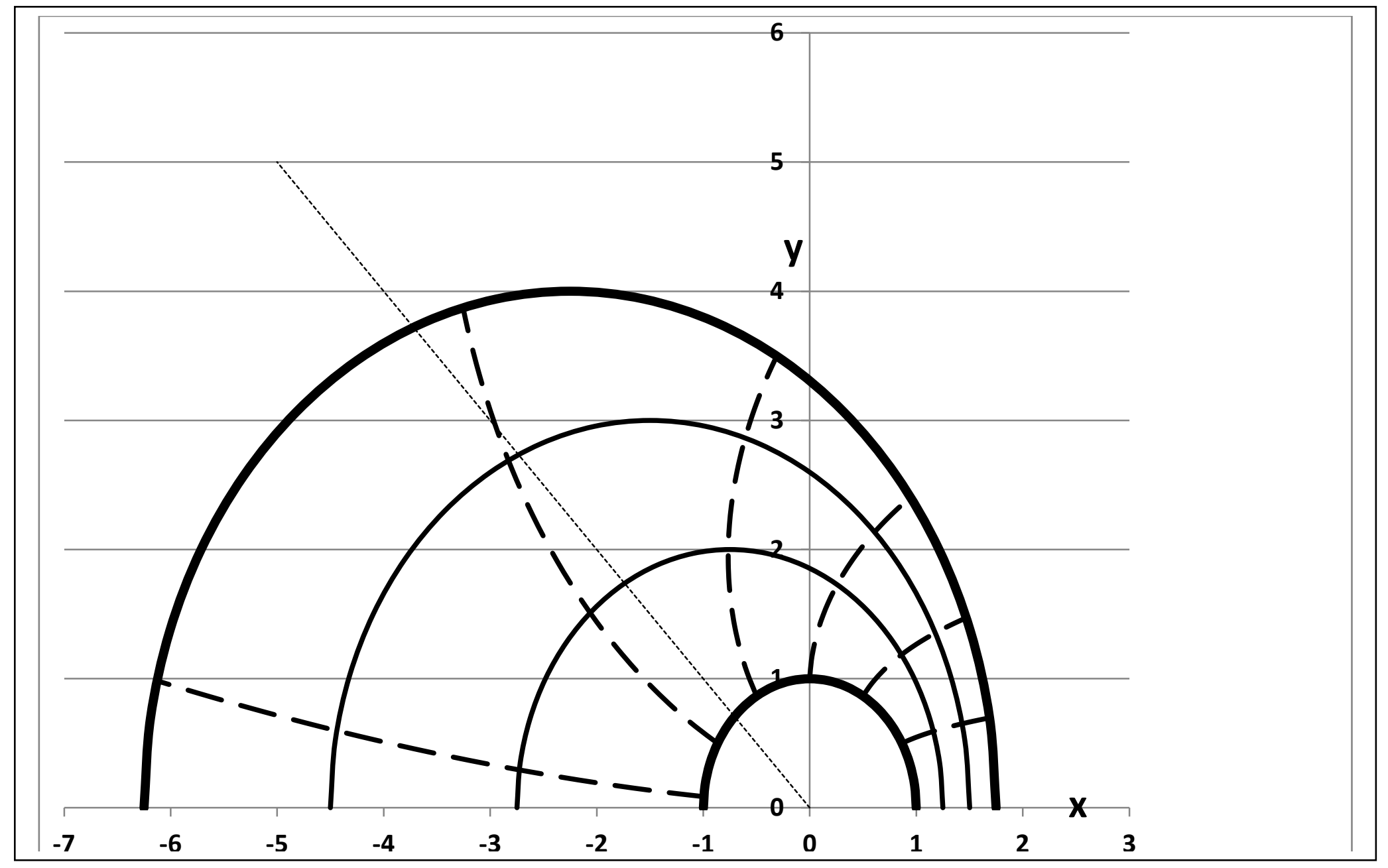

Figure 2 Constant $X$-lines and corresponding $\psi_{1}$ lines, projected onto the horizontal plane $\mathrm{X}$-lines represent the paths of azimuthal principal stress $\sigma_{2}$. The $\psi_{1}$ lines are the paths of radial stress $\sigma_{1}$ arbitrary scale; radii are full lines; $\psi_{1}$ lines are heavy, dashed lines; the dotted line is a line of constant $\varepsilon_{1}$ 


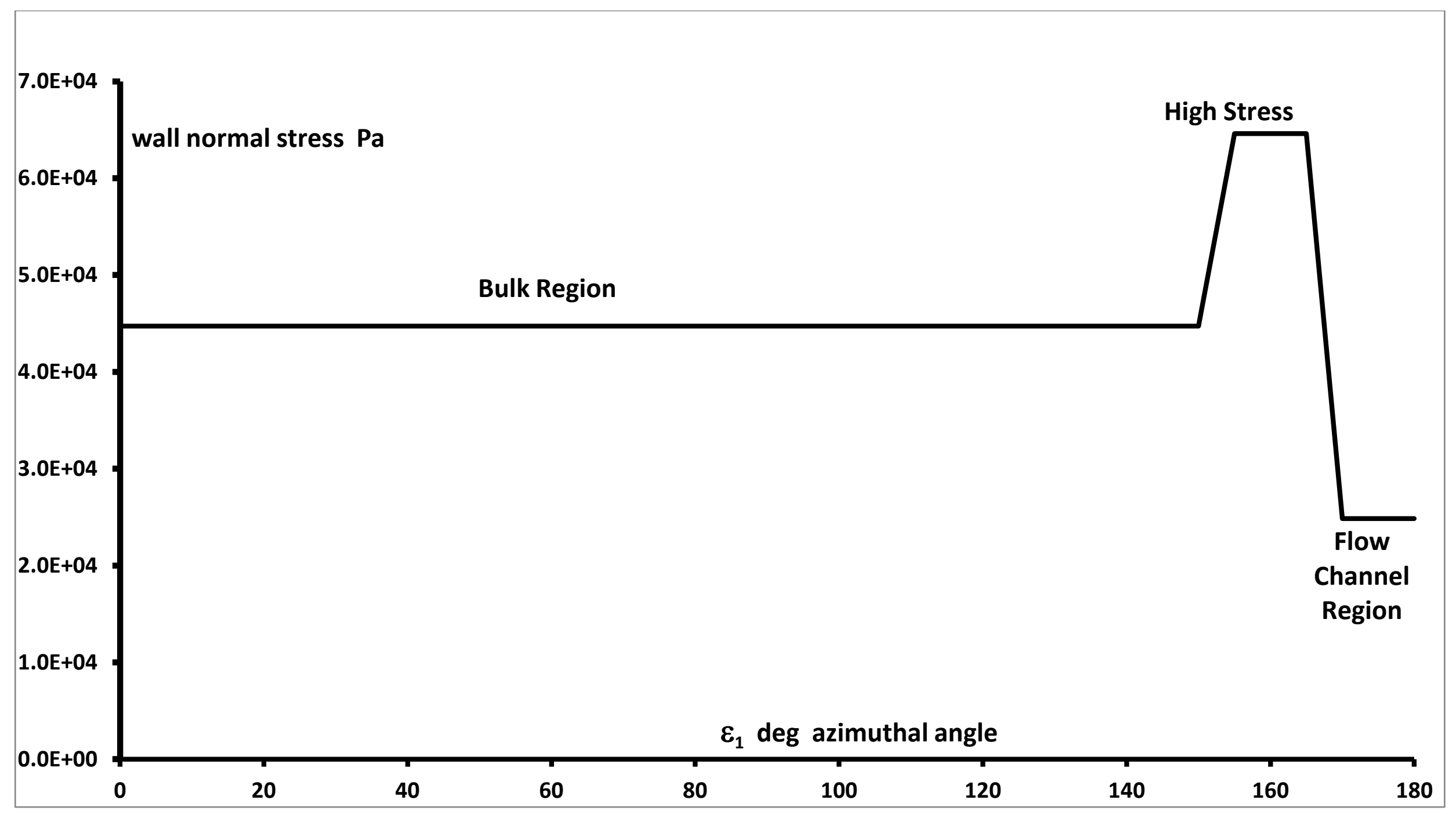

Figure 3 Wall normal stress data calculated by Eurocode 1 for a flow channel next to the silo wall 


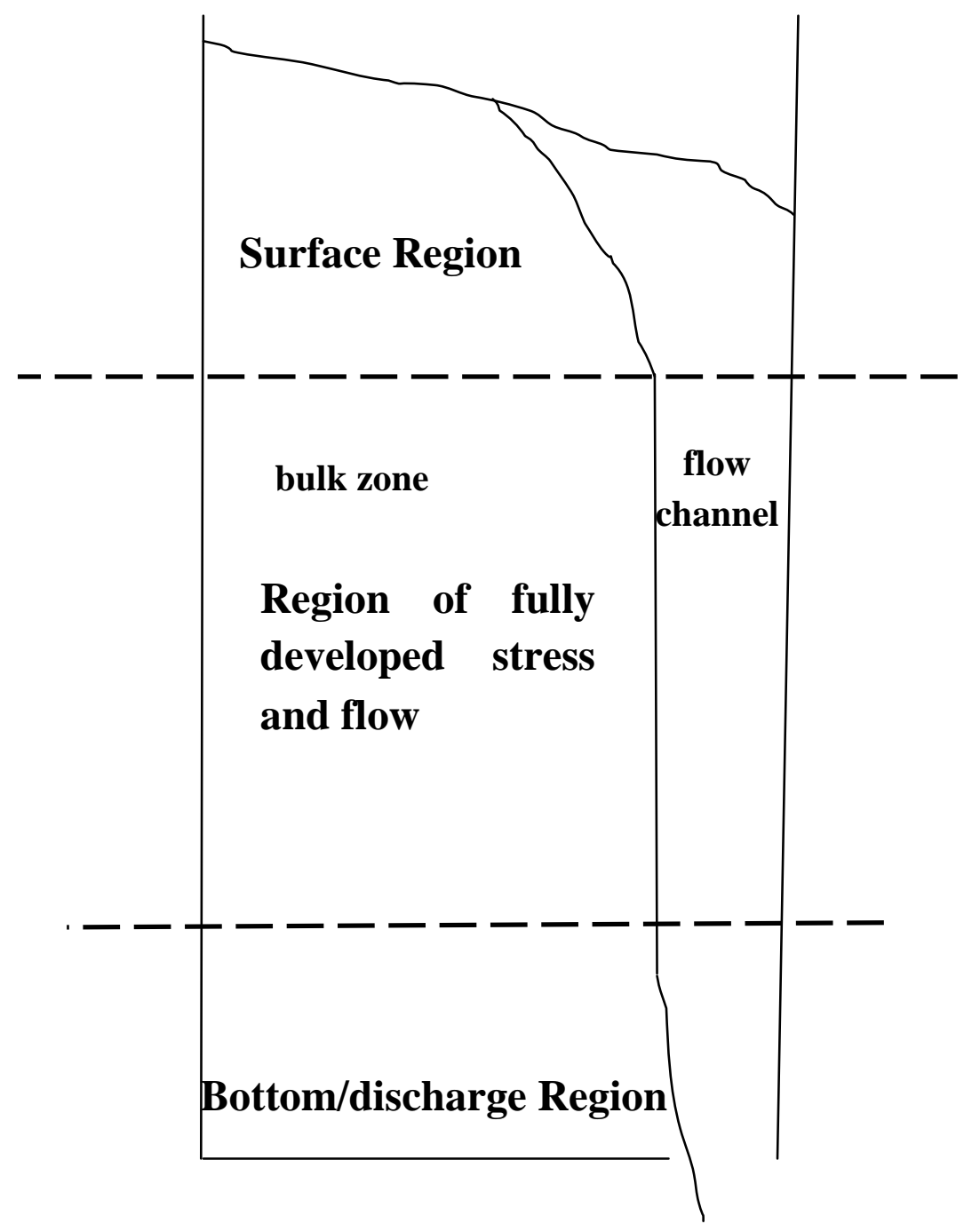

Figure 4 The conventional picture of a wall flow channel 


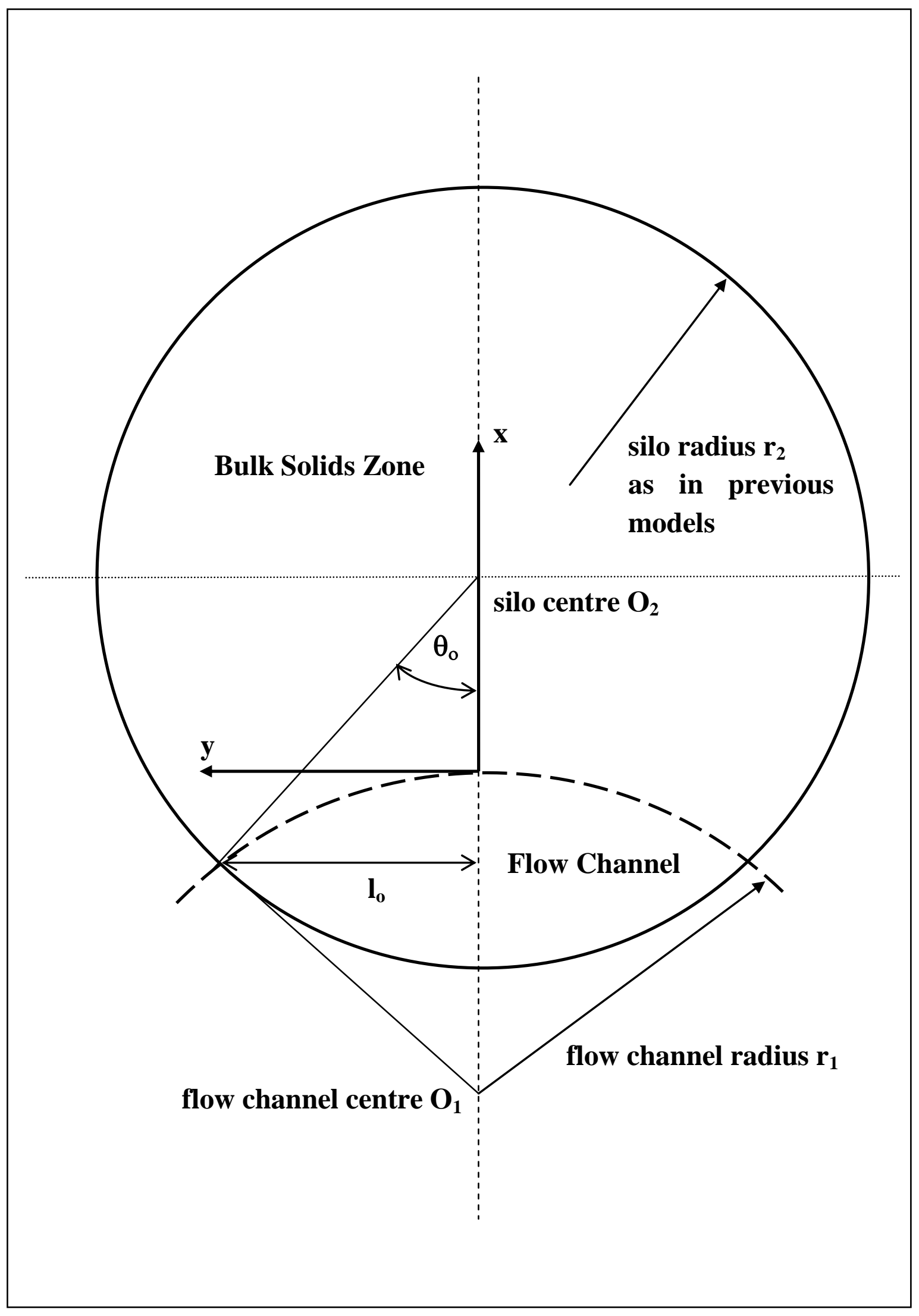

Figure 5 Cross-section of the region of fully developed stress, showing the flow channel, which is circular in section and crosses the silo wall at right-angles 


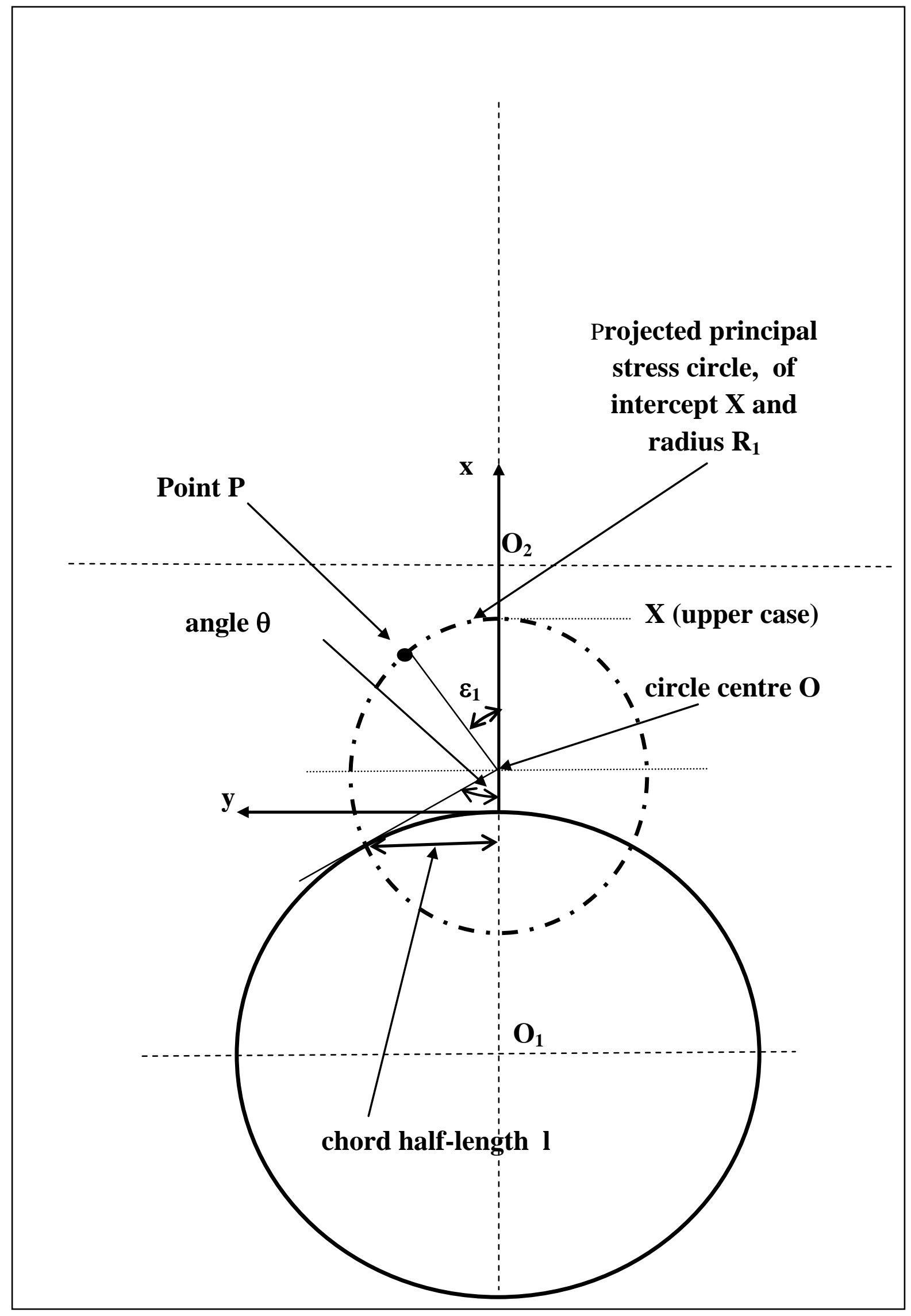

Figure 6 General azimuthal projected principal stress circle $\mathbf{X}$ 


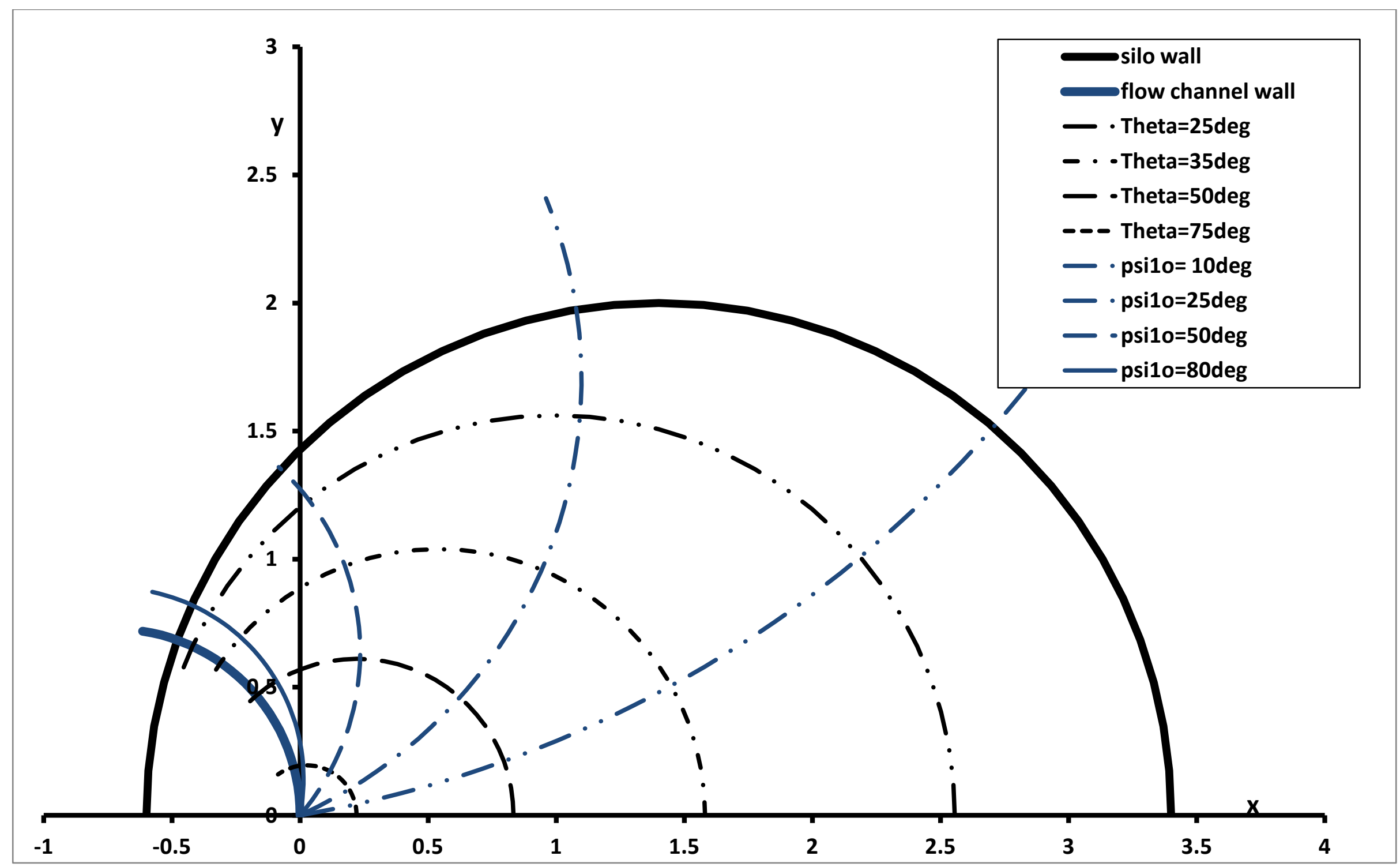

Figure $7 X$ and $\psi_{1}$ lines projected onto a horizontal plane. $r_{2}=2 ; \theta_{0}=20^{\circ} ; l_{0}=0.684 \mathrm{~m} ; r_{1}=0.728 \mathrm{~m}$ 


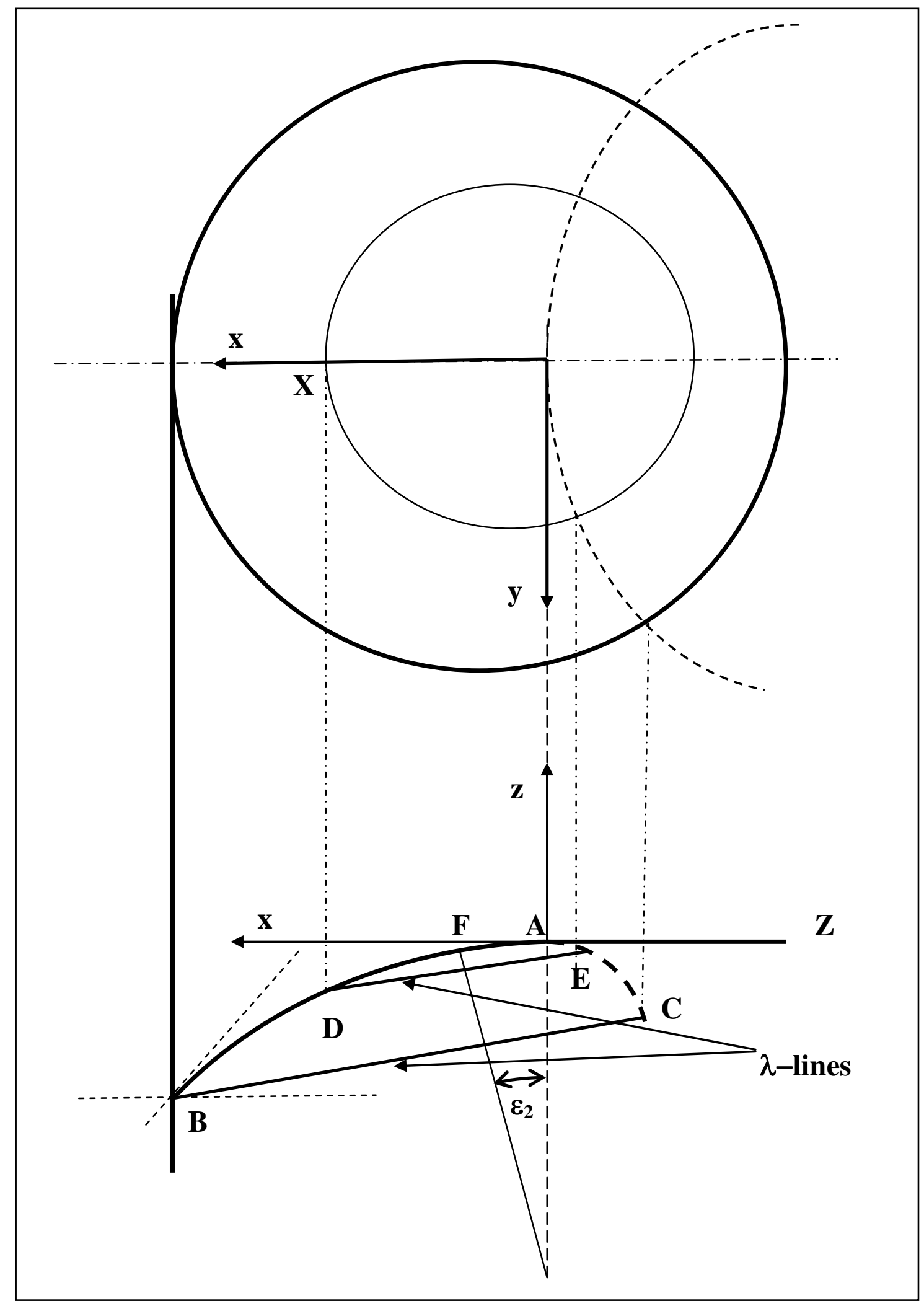

Figure 8 Plan view(top) and side view from the direction of the y-axis of the principal stress cap surface. 


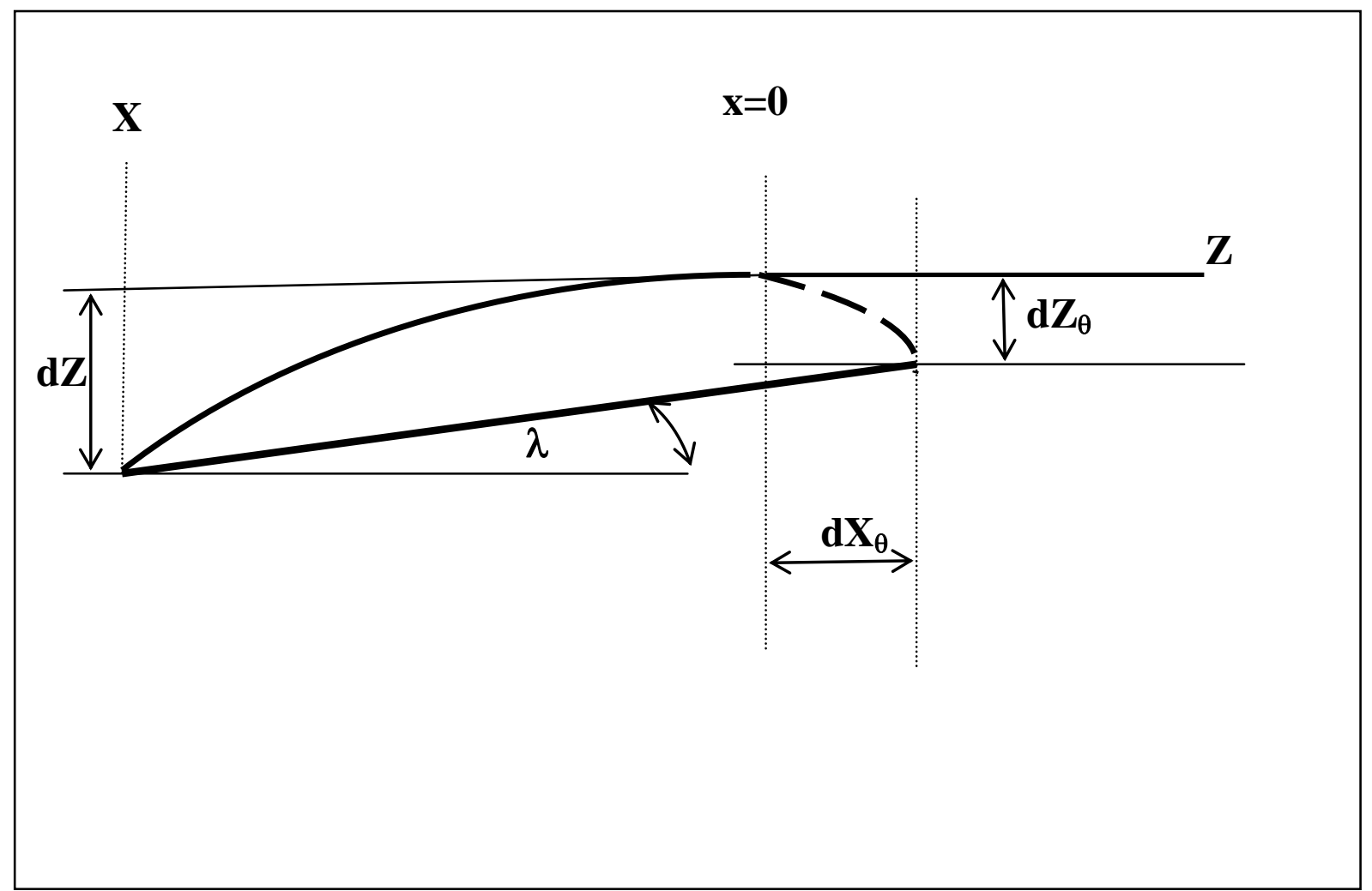

Figure 9 View of the stress cap surface and $\lambda$-line at $X$, seen from the $y$-axis. The calculation of $\lambda$, showing offsets in $X$ and $Z$ associated with the flow channel geometry. 
Eurocode 1 surface plots

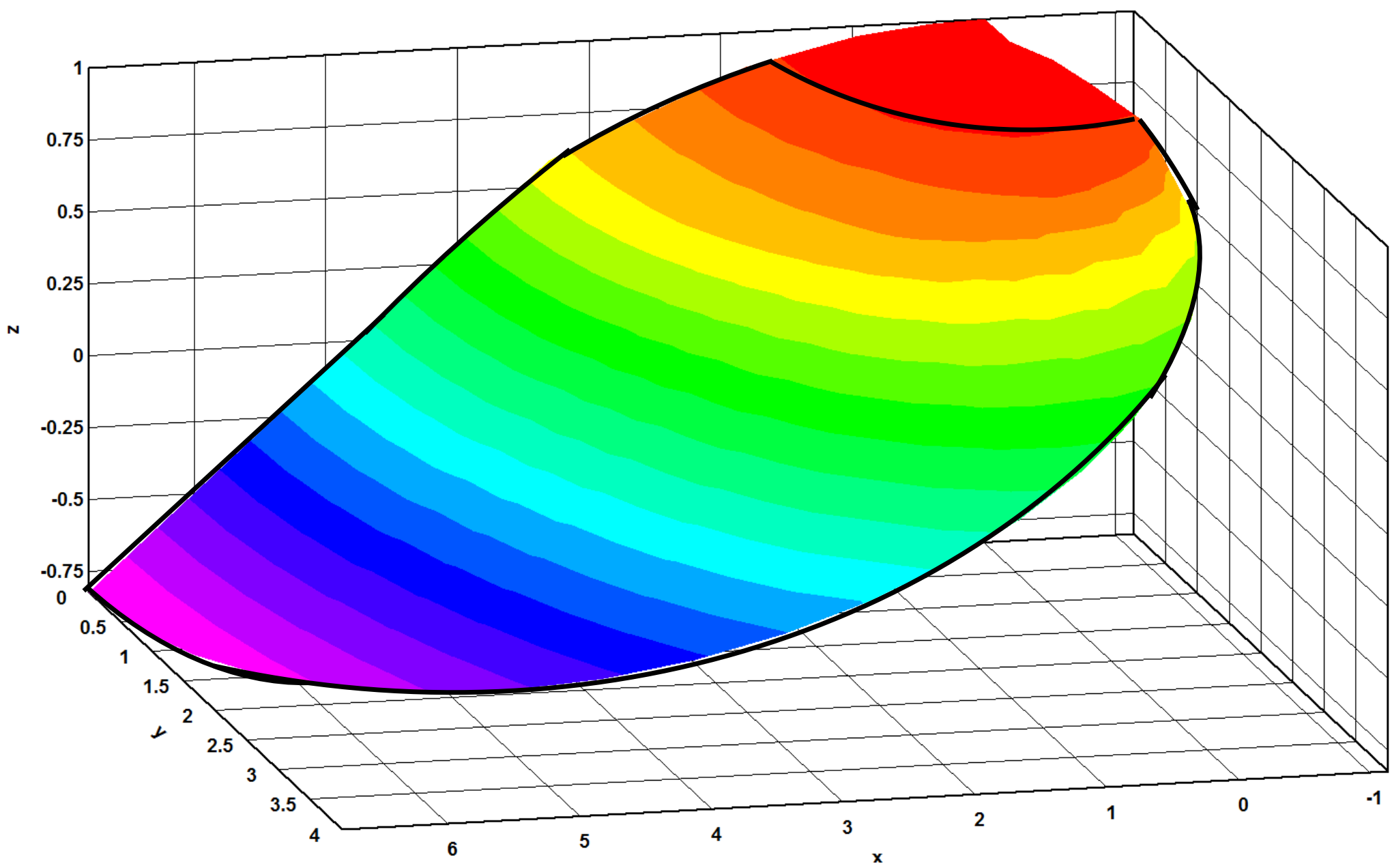

Figure 10 3-d plot of a principal stress cap half-surface for the Eurocode 1 model. $r_{2}=4 \mathrm{~m} ; \beta_{\mathrm{w}}=30^{\circ} ; \beta_{\mathrm{c}}=0$ 


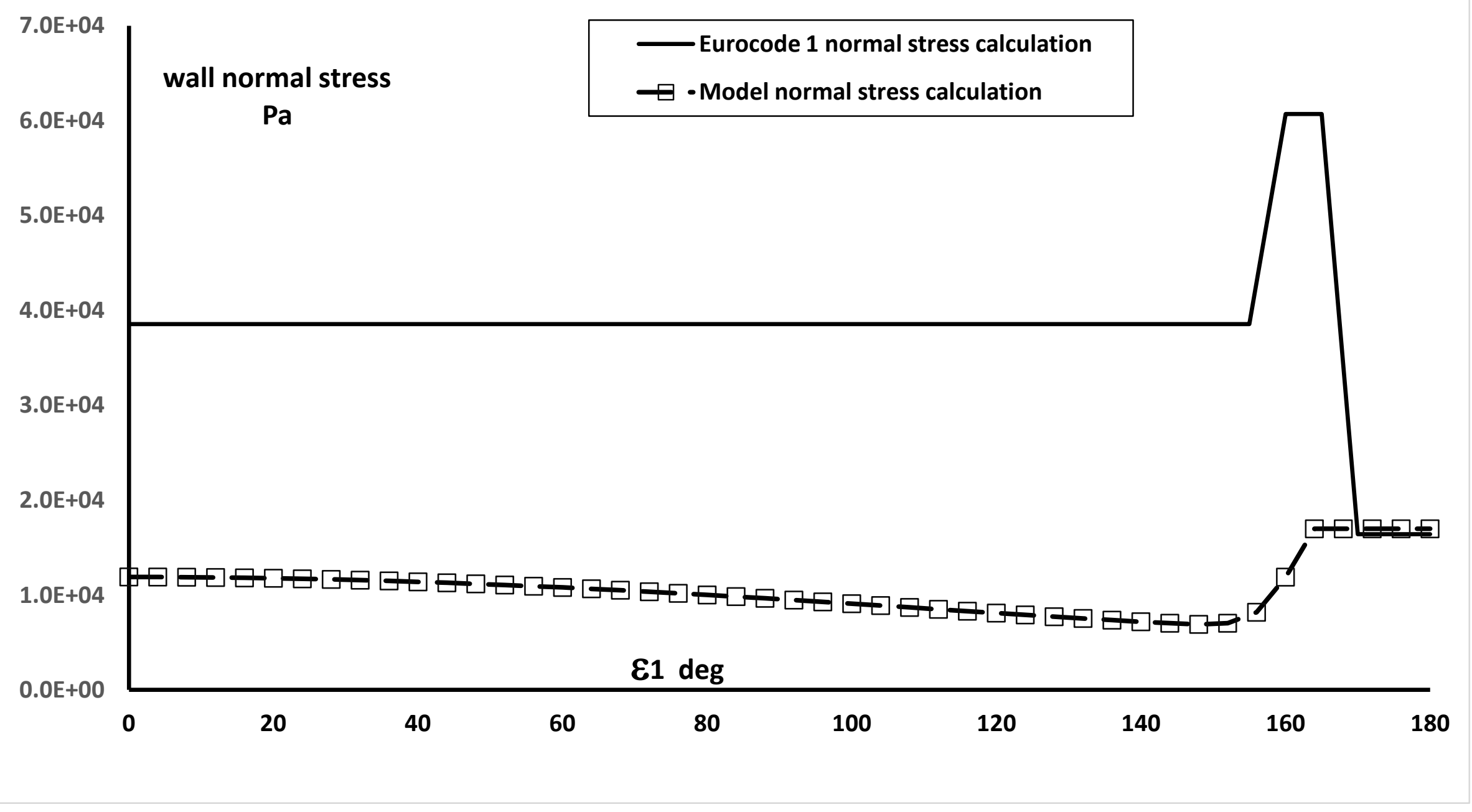

Figure 11 Comparison of wall normal stress for the two region model and Eurocode 1 calculations. $r_{2}=4 \mathrm{~m} ; \beta_{\mathrm{w}}=10^{\circ} ; \mathrm{l}_{0}=1.2 \mathrm{~m} ; \mathrm{r}_{1}=1.26 \mathrm{~m} ; \mu=0 ; \mu_{\mathrm{w}}=0.2 ; \mathrm{Kw}=0.3$ 


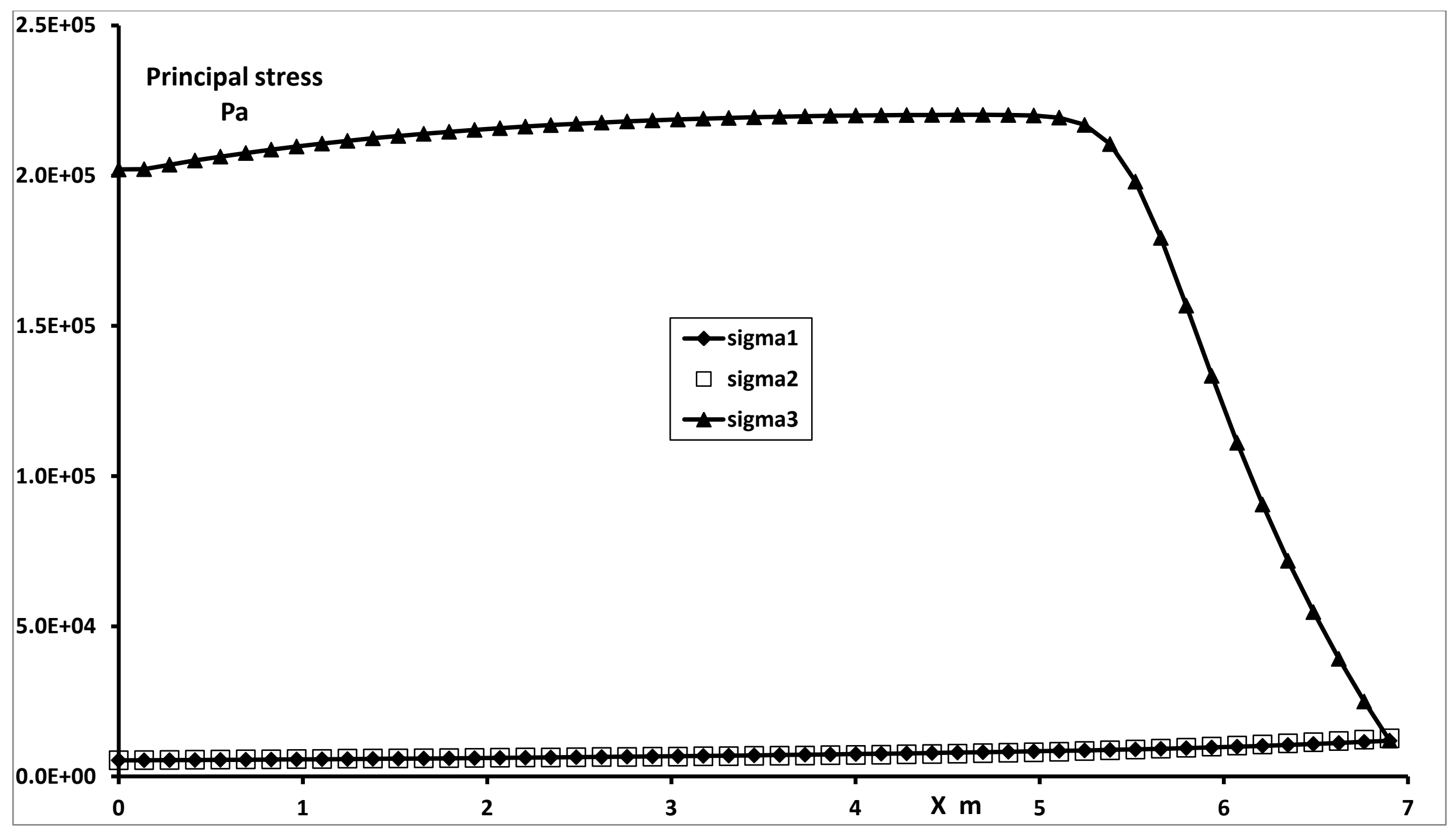

Figure 12 Variation in principal stresses along the $\mathrm{x}$-axis; $\varepsilon_{1}=0$. Condition as in the simulation in Figure 9 


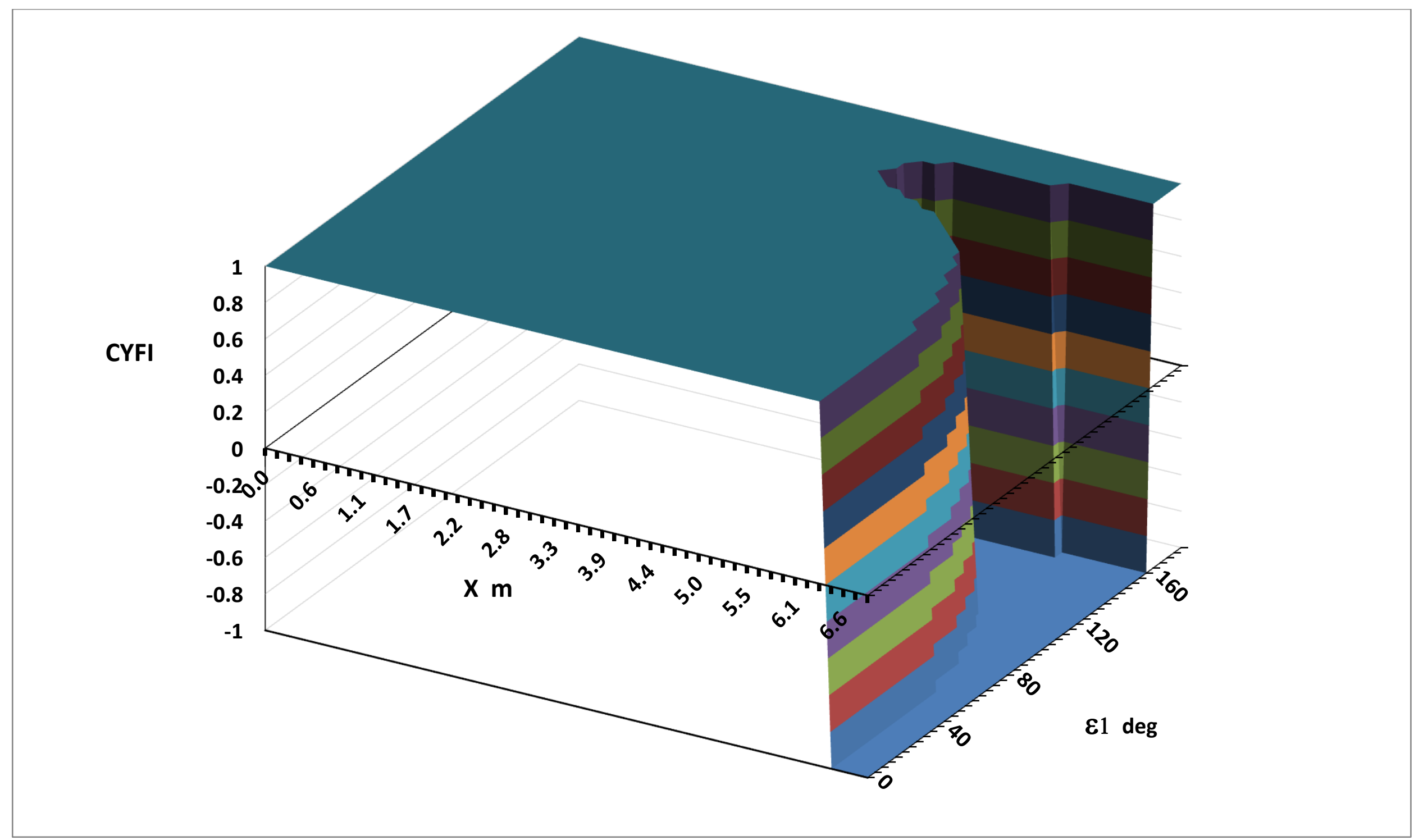

Figure 13 Conical Yield Function Index in $X-\varepsilon_{1}$ space for conditions in Figure 9 


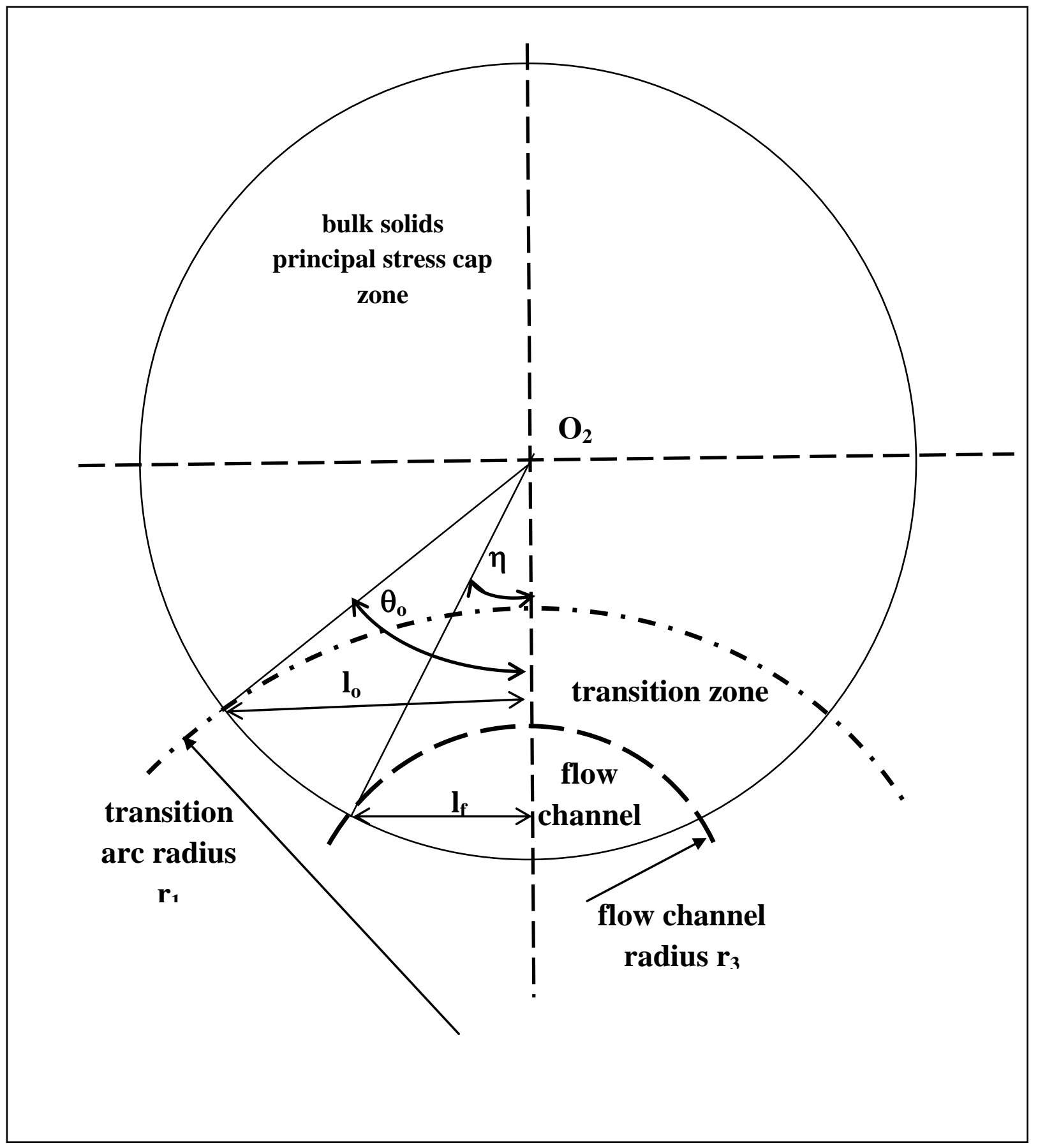

Figure 14 Three zone model for a flow channel in the vicinity of the silo wall, showing the transition zone 


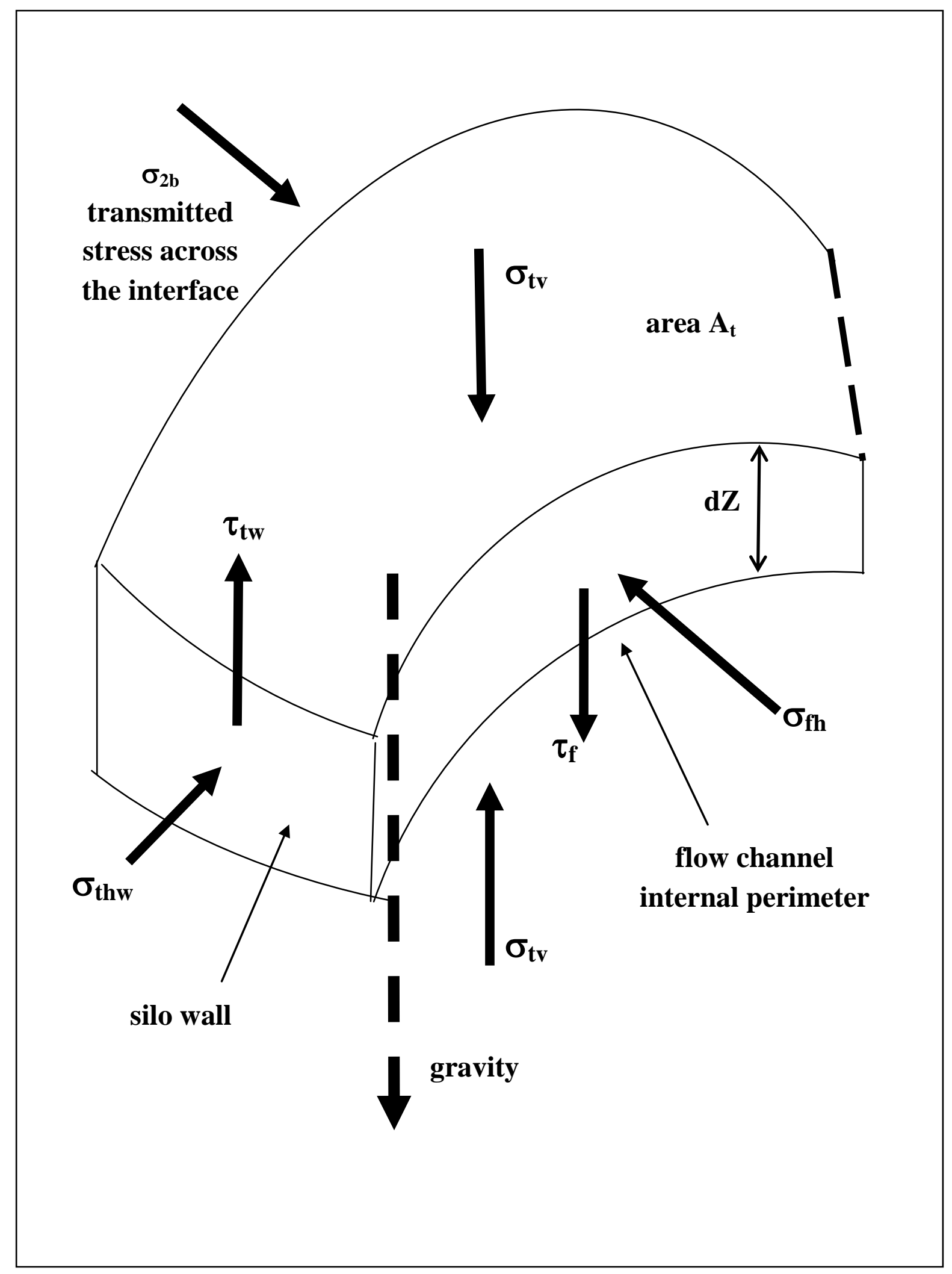

Figure 15 Force balance on the transition zone 



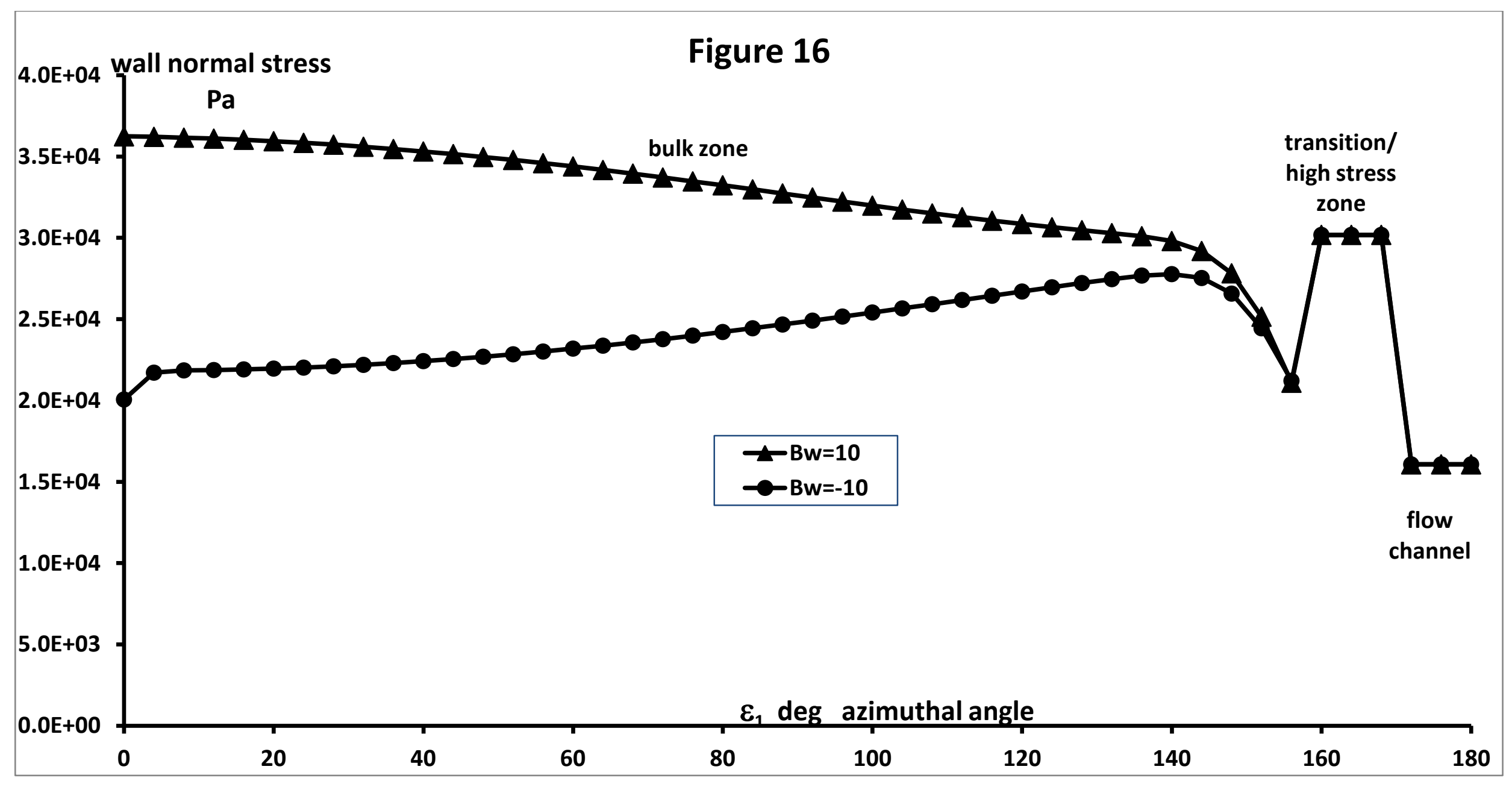

Figure 16 General wall normal stress response for the three-zone model $r_{2}=4 \mathrm{~m} ; \mathrm{l}_{\mathrm{o}}=1.6 \mathrm{~m} ; \mathrm{l}_{\mathrm{f}}=1.42 \mathrm{~m} ; \mu=-0.1 ; \mu_{\mathrm{w}}=0.25 ; \mu_{\mathrm{tw}}=0.125 ; K_{\mathrm{f}}=3 ; K_{\mathrm{t}}=2 ; K_{\mathrm{tb}}=1$ $\beta_{\mathrm{w}}=10$ and $\gamma_{1}=-1$ or $\beta_{\mathrm{w}}=-10$ and $\gamma_{1}=1$ 


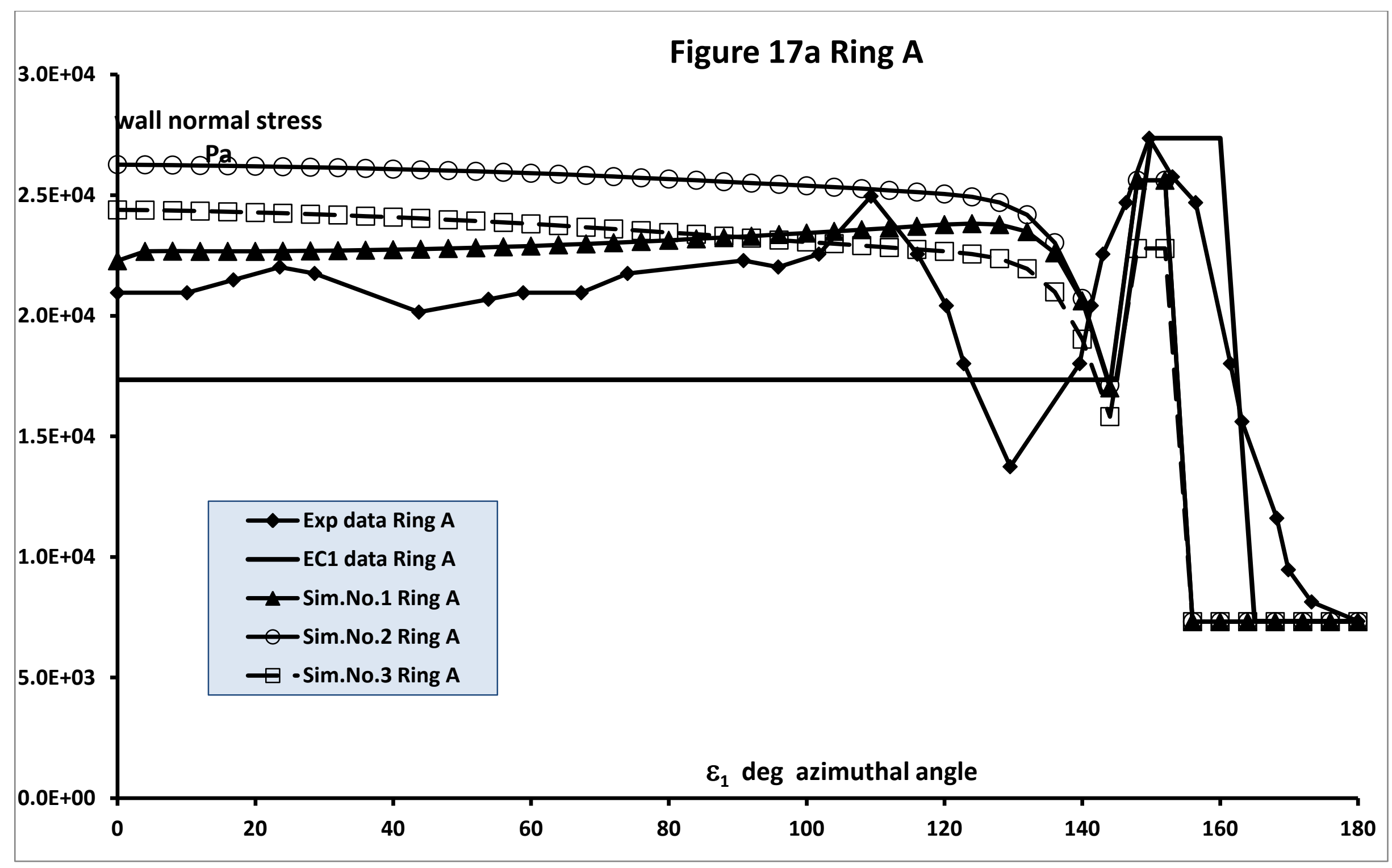




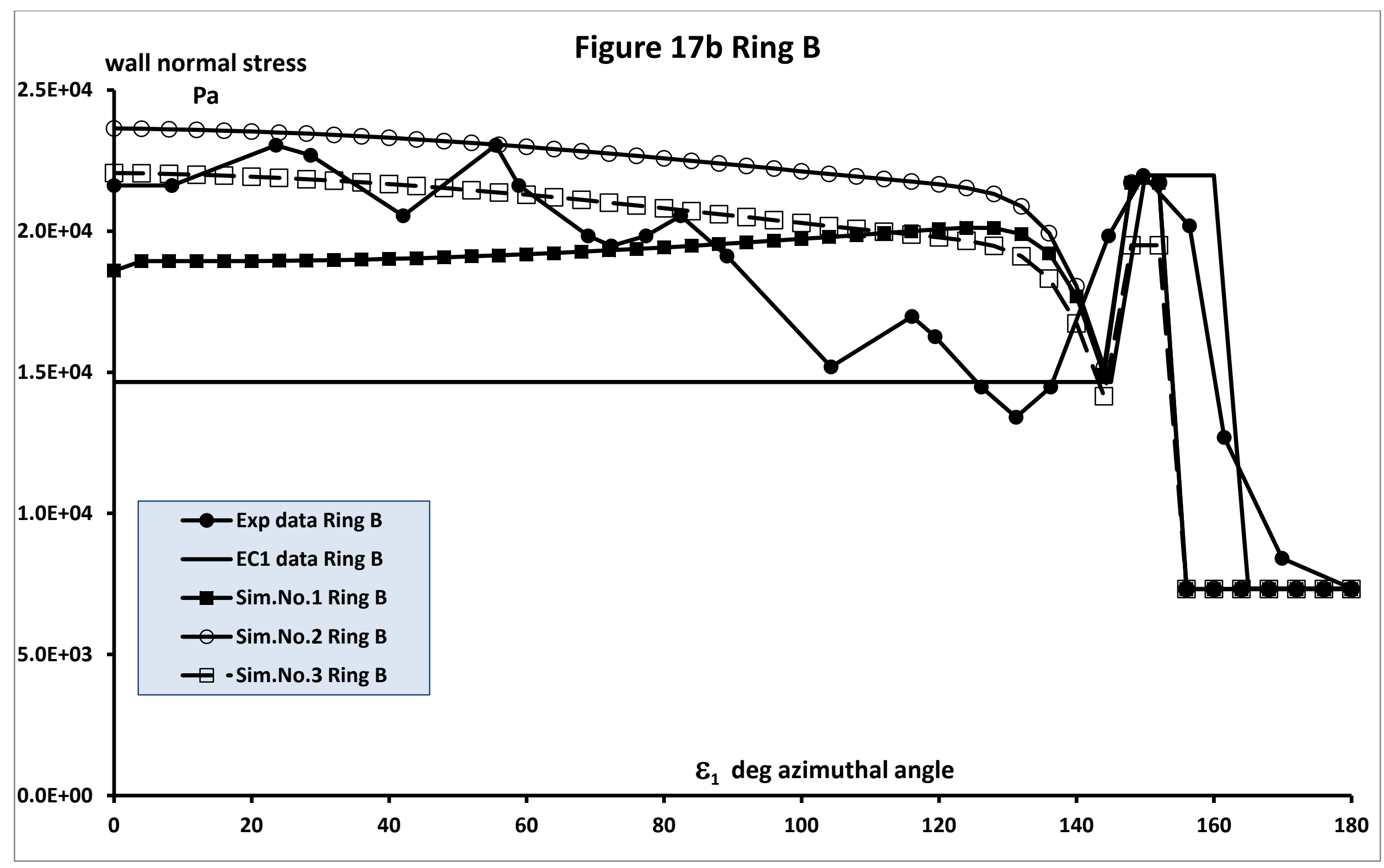




\section{Figure 75c Ring C}

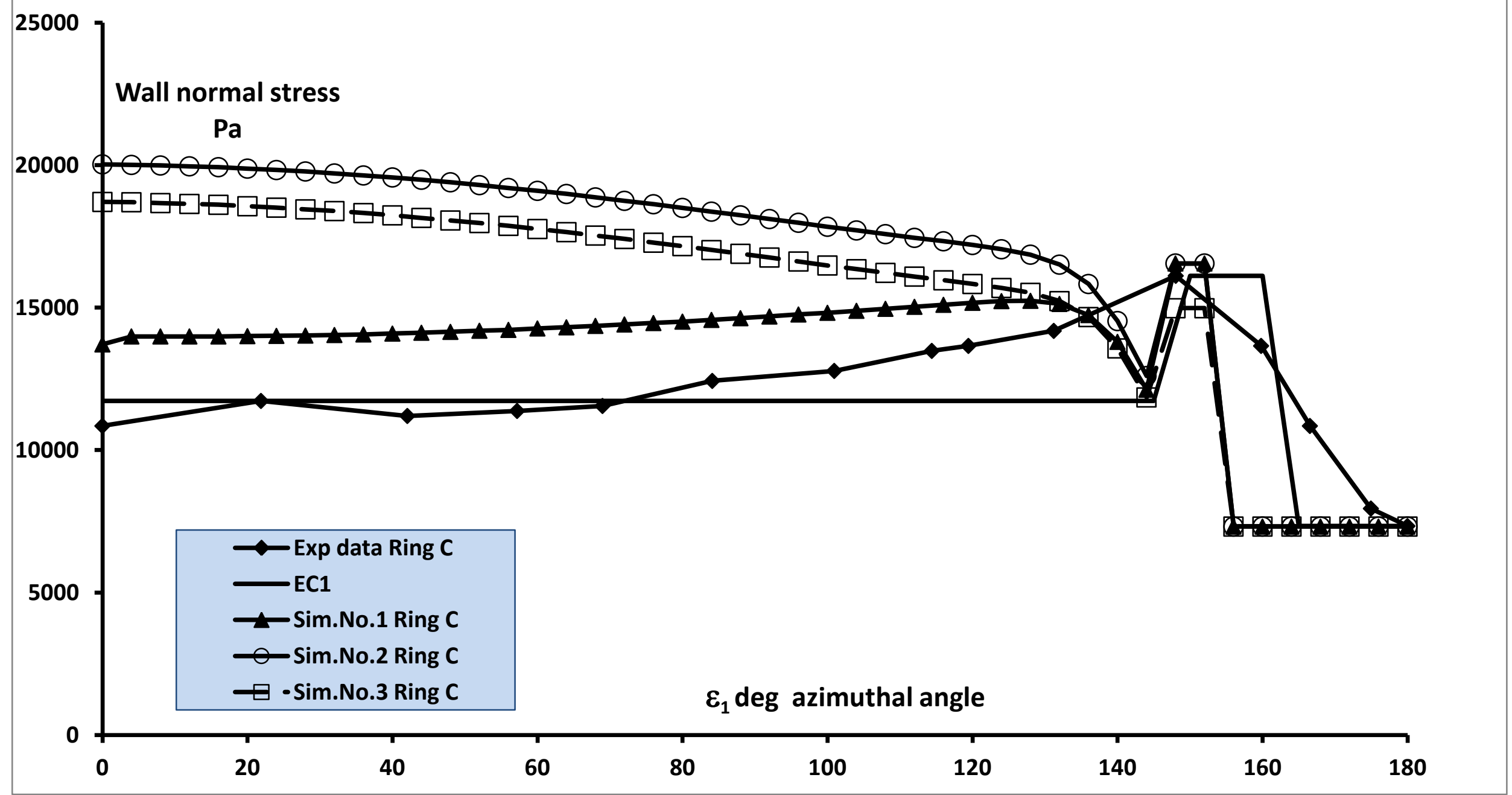




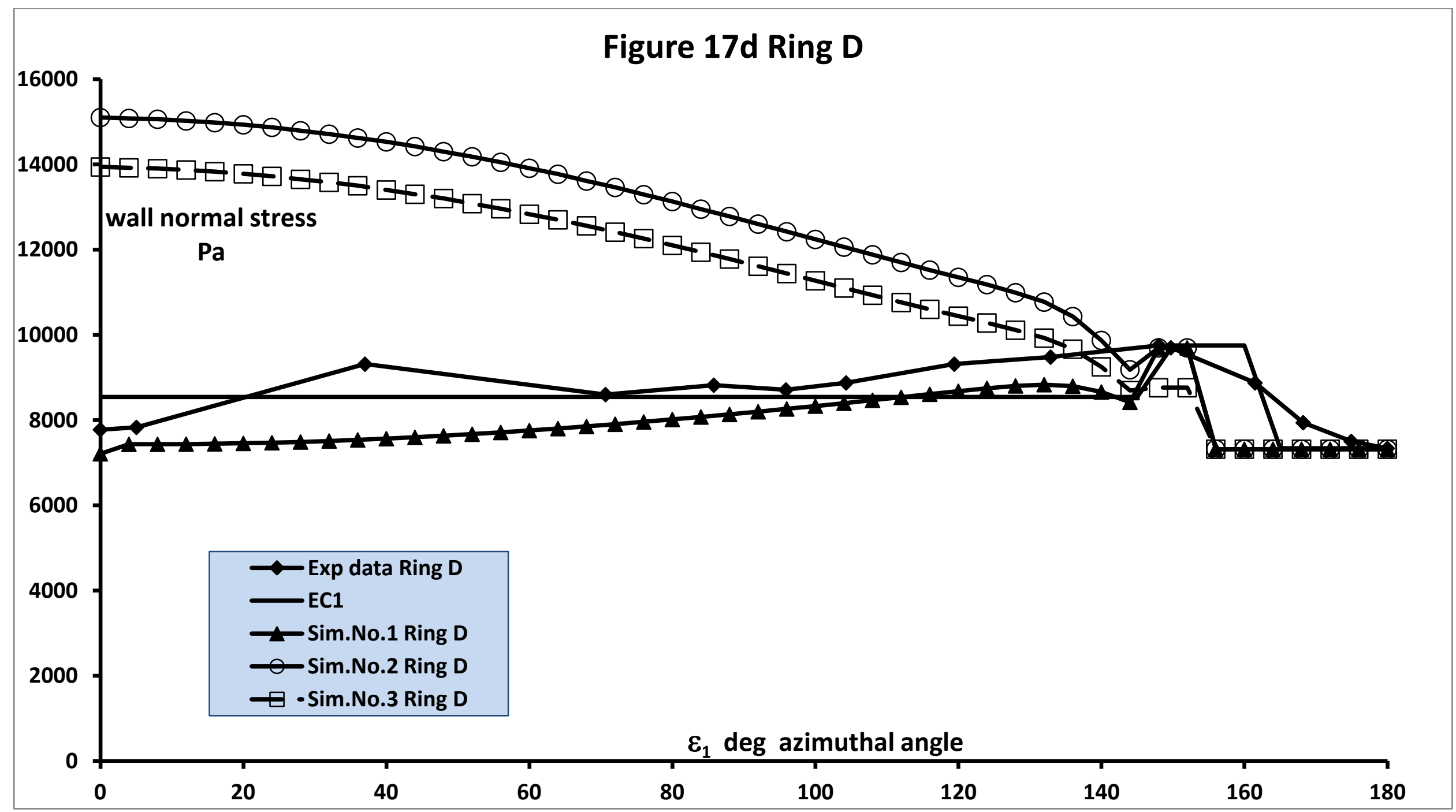


Figure 17 Comparison of Chen et al data(2007) with model predictions. A "best-fit" of data to the model.

Figure 17a Ring A, depth 8m

Figure 17b Ring B, depth $6.5 \mathrm{~m}$

Figure 17c Ring C, depth 5m

Figure 17d Ring D, depth 3.5m

See Table 1 for silo and material properties

See Table 2 for model parameters 